\title{
On the aerodynamic forces on heaving and pitching airfoils at low Reynolds number
}

\author{
M. Moriche, O. Flores, M. García-Villalba \\ Departamento de Bioingeniería e Ingeniería Aeroespacial \\ Universidad Carlos III de Madrid \\ 28911 Leganés, SPAIN
}

\begin{abstract}
The influence that the kinematics of pitching and heaving $2 \mathrm{D}$ airfoils have on the aerodynamic forces is investigated using Direct Numerical Simulations and a force decomposition algorithm. Large amplitude motions are considered (of the order of one chord), with moderate Reynolds numbers and reduced frequencies of order $O(1)$, varying the mean pitch angle and the phase shift between the pitching and heaving motions. Our results show that the surface vorticity contribution (viscous effects) to the aerodynamic force is negligible compared to the contributions from the body motion (fluid inertia) and the vorticity within the flow (circulation). For the range of parameters considered here, the latter tends to be instantaneously oriented in the direction normal to the chord of the airfoil. Based on the results discussed in the paper, a reduced order model for the instantaneous aerodynamic force is proposed, taking advantage of the force decomposition and the chord-normal orientation of the contribution from vorticity within the flow to the total aerodynamic force. The predictions of the proposed model are compared to those of a similar model from the literature, showing a noticeable improvement on the prediction of the mean thrust, and a smaller improvement on the prediction of mean lift and the instantaneous force coefficients.
\end{abstract}

\section{Introduction}

Driven by the recent development of Micro Air Vehicles (MAVs), unsteady aerodynamics of flapping wings has attracted the interest of the scientific community during the past decades. MAVs operating conditions are similar to those in which insects and small birds fly: the Reynolds (Re) number of the flow is about 10 to $10^{4}$ and the motion of the wings is characterized by moderate frequencies and high amplitudes [Shyy et al., 2013]. The maneuverability and performance of these animals is outstanding and, therefore, a deep insight in the aerodynamics of flapping flight is essential to improve the design of MAVs. In particular, it is important to understand how aerodynamic forces are generated and to use this knowledge for the improvement of simplified force models. There is a broad literature on the aerodynamics of flapping wings as recently reviewed by several authors Rozhdestvensky and Ryzhov, 2003, Platzer et al., 2008, von Ellenrieder et al., 2008, Shyy et al., 2010, 2013.

In order to improve the understanding of flapping wing aerodynamics, scientists have typically studied simplified configurations. Numerous authors have studied the problem of a 2D airfoil in pure heaving motion, in which the airfoil oscillates vertically with a zero angle of attack [Jones and Platzer. 1997. Wang, 2000, Lewin and Haj-Hariri, 2003, Lua et al., 2007, Wei and Zheng, 2014, Choi et al. 2015. This simplified configuration is still a rich model where some of the main features of flapping flight are present, for example, the leading and trailing edge vortices. The leading edge vortex (LEV) has been identified as the main lift enhancing mechanism of flapping wings Ellington et al., 1996. In fixed wing aerodynamics, the generation of an LEV produces a high lift plateau for a short time span followed by a sudden drop of the aerodynamic force [Carr, 1988. This process is known as dynamic stall. Conversely, flapping wings take advantage of the high lift generated during the formation of 
the LEV by consecutively generating an LEV in each stroke. With this cyclic mechanism, the wing experiences the high transient lift from the generation of an LEV and avoids entering in the dynamic stall region. Wang [2000] studied a heaving airfoil at $R e=1000$ by direct numerical simulations (DNS). She found that tuning the motion parameters so that the time scales of the motion and the LEV are similar, results in an optimal performance of the airfoil in terms of the aerodynamic forces. In an extensive numerical analysis on heaving airfoils at $R e=500$, Lewin and Haj-Hariri 2003 explain how the interaction between the LEV and the trailing edge vortex (TEV) influences the propulsive efficiency of the airfoil. With the introduction of non-zero angle of attack, the airfoil may generate both thrust and lift. Heaving and pitching airfoils have also been studied extensively [Anderson et al., 1998, Ramamurti and Sandberg, 2001, Read et al., 2003, Ashraf et al., 2011, Baik et al., 2012, Widmann and Tropea, 2015]. Pitching modifies the flow around the airfoil and can, for some combinations of the motion parameters, increase the net value of thrust and the propulsive efficiency.

In order to obtain a deeper insight in the generation of forces by flapping airfoils, several authors have proposed various methods to decompose the total aerodynamic force in different contributions Chang, 1992, Noca et al., 1999, Wu et al., 2005. These methods differ in the surface and volume integrals they involve, although it is possible to establish mathematical relations between them, see Appendix in Wang et al. 2015]. From a practical point of view it seems desirable to have a method where the terms are easy to compute and have a clear physical meaning. Indeed, Wang et al. 2015] proposes an approximate 'simple lift formula' decomposition, and compares it to the methods of Noca et al. [1999] and Wu et al. [2005], finding that added mass and circulatory effects were the main contributions to the aerodynamic force. This result is in agreement with the recent work of MartínAlcántara et al. [2015], who employed the decomposition method proposed by Chang [1992] on 2D DNS data of a heaving airfoil at $R e=500$. Martín-Alcántara et al. [2015] also observed that the contribution to the aerodynamic force of vortical structures which are a few chords away from the airfoil is negligible.

The analysis of the aerodynamic forces in terms of their various contributions might be used to predict the aerodynamic forces on a flapping wing from its geometry and kinematics. New models to estimate the aerodynamic forces can be generated and, also, existing models can be improved. Many such models exist since the pioneering work of Wagner 1925] and Theodorsen [1949, among others, and they have been recently reviewed by Ansari et al. [2006] and Taha et al. [2012]. For small amplitude motions at high $R e$ there is a complete theory based on potential flow that predicts the aerodynamic forces produced on a thin airfoil [Wagner, 1925, Theodorsen, 1949]. A less restrictive approach is provided by unsteady vortex lattice methods (UVLM). These methods present no restriction regarding the motion of the airfoil nor its geometry Long and Fritz, 2004. However, since UVLM are also based on potential flow theory, they are not able to capture leading edge separation. Therefore, these methods need modifications to include the contribution of LEVs, as for example proposed by Ansari et al. 2006]. Despite the fact that modified UVLM are computationally inexpensive compared to other methods like DNS, their cost might still be too high to predict the forces on-the-fly in the small processors installed in MAVs. This motivates the development of even simpler models, like the model proposed by Dickinson et al. [1999]. This model is quasi-steady and uses algebraic expressions for the drag and lift coefficients as a function of the instantaneous angle of attack, capturing the effect of the LEV in the force coefficients. The algebraic expressions in the model were calibrated using experimental data. Another quasi-steady model was developed by Pesavento and Wang [2004] working with free falling plates. They estimated the total aerodynamic force by separately modelling the added mass, circulatory and viscous effects. In their work they propose an algebraic model for the circulation of an airfoil in which high angles of attack and rotational effects are included. A similar approach was followed by Taha et al. [2014] but including the effect of the wake using the Wagner function and neglecting viscous effects.

With the aim of contributing to the improvement of simplified models for the aerodynamic forces, in this work we analyze DNS data of heaving and pitching airfoils, using the force decomposition 


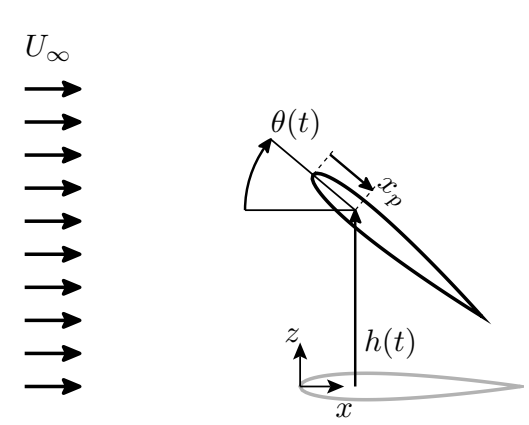

(a)

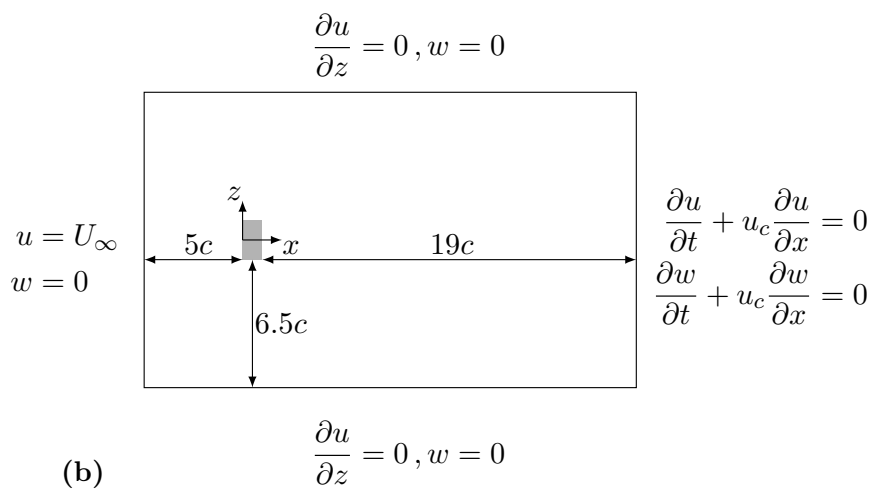

(b)

Figure 1: a) Sketch of the heaving and pitching motion of the airfoil. b) Sketch of the computational domain. $u$ and $w$ are the velocities in the $x$ and $z$ directions, respectively and $u_{c}$ is the convective velocity. The airfoil is represented in red and the region in which the motion takes place in light gray.

method proposed by Chang [1992]. Based on this analysis, we propose and test a simple model for the aerodynamic forces using the aforementioned force decomposition, and elements of the model proposed by Pesavento and Wang 2004]. The database used in the present paper was first introduced in Moriche et al. [2015, and some of the cases of this database were analyzed in Moriche et al. 2016], with emphasis on the development of 3D instabilities.

The paper is organized as follows. In section 2 the details of the numerical method, computational setup and force decomposition method are presented. In section 3 the complete database is described in terms of mean and rms total aerodynamic force coefficients. In section 4 the force decomposition method is applied to a reference case and the main contributions to the force are modelled. After that, we extend the analysis to a subset of cases from the database in section 5 , and to the whole database in section 6. Finally, some conclusions are provided in section 7 .

\section{Numerical method}

We present DNS of the flow around a symmetric airfoil NACA 0012 in heaving and pitching motion. The Reynolds number of the flow based on the airfoil chord $c$ and the free stream velocity $U_{\infty}$ is $R e=c U_{\infty} / \nu=1000$, where $\nu$ is the kinematic viscosity of the fluid. The simulations have been performed using TUCAN, a second order finite differences code that solves the Navier-Stokes equations for an incompressible flow Moriche, 2017]. The presence of the body is modelled by the direct forcing immersed boundary method proposed by Uhlmann [2005].

The prescribed heaving and pitching motion of the airfoil is given by

$$
\begin{aligned}
& h(t)=h_{0} \cos (2 \pi f t), \\
& \theta(t)=\theta_{m}+\theta_{0} \cos (2 \pi f t+\varphi),
\end{aligned}
$$

where $h_{0}$ and $\theta_{0}$ are the heaving and pitching amplitude, respectively, $\theta_{m}$ is the mean pitch value, $\varphi$ is the phase shift between the heaving and pitching motion and $f$ is the frequency of the motion. The pitching motion is a rotation around a point located at a distance $x_{p}$ from the LE, as it can be observed in the sketch shown in figure 1 1 a. The set of non-dimensional parameters which define the problem $\left(h_{0} / c, \theta_{m}, \theta_{0}, \phi, 2 \pi f c / U_{\infty}, x_{p} / c, \rho U_{\infty} / \nu\right)$ results in a large parametric space, so we only vary two of them. The Reynolds number is $R e=1000$ and the pivoting point is located at the quarter of the chord $\left(x_{p}=c / 4\right)$. The heaving amplitude is $h_{0}=c$, the pitching amplitude is $\theta_{0}=30^{\circ}$ and the 
reduced frequency ${ }^{1}$ is $k=2 \pi f c / U_{\infty}=1.41$, resulting in a period of oscillation $T=4.44 c / U_{\infty}$. The effect of the mean pitch angle and the phase shift between heaving and pitching are explored, varying $\theta_{m}$ in the range $0^{\circ}$ to $20^{\circ}$ in steps $\Delta \theta_{m}=10^{\circ}$ and $\varphi$ from $30^{\circ}$ to $130^{\circ}$ in steps $\Delta \varphi=20^{\circ}$, resulting in a database of 18 simulations.

All simulations are performed in a computational domain of dimensions $25 c \times 15 c$ in the streamwise and vertical directions, respectively. The resolution used in this study is 128 points per chord, yielding a total of $3200 \times 1920$ grid points in the streamwise and vertical directions, respectively. This resolution has been selected based on a grid refinement study for a NACA 0012 at $R e=1000$ set in heaving and pitching motion (see appendix A). The free stream condition is modelled by an inflow velocity $U_{\infty}$ at the inlet boundary, located $5 c$ upstream of the airfoil's leading edge. The outflow is modelled with an advective boundary condition at the outlet, located $19 \mathrm{c}$ downstream of the airfoil's trailing edge. A free slip boundary condition is imposed at the lateral boundaries (see figure 1 $1 \mathrm{~b}$ ).

The total aerodynamic force $\vec{F}$ is decomposed using the algorithm proposed by Chang [1992] and recently used by Martín-Alcántara et al. [2015]. The total aerodynamic force components in the streamwise $(x)$ and vertical $(z)$ directions are expressed as

$$
\begin{aligned}
& F_{x}=-\rho \int_{S} \frac{\phi_{x}}{U_{\infty}} \frac{\partial \vec{u}}{\partial t} \cdot \vec{n} \mathrm{~d} S+\frac{\rho}{2} \int_{S}|\vec{u}|^{2} \vec{n} \cdot \vec{e}_{x} \mathrm{~d} S-\rho \int_{V}(\vec{u} \times \vec{\omega}) \cdot \frac{\nabla \phi_{x}}{U_{\infty}} \mathrm{d} V+\mu \int_{S}(\vec{\omega} \times \vec{n}) \cdot\left(\frac{\nabla \phi_{x}}{U_{\infty}}+\vec{e}_{x}\right) \mathrm{d} S, \\
& F_{z}=-\rho \int_{S} \frac{\phi_{z}}{U_{\infty}} \frac{\partial \vec{u}}{\partial t} \cdot \vec{n} \mathrm{~d} S+\frac{\rho}{2} \int_{S}|\vec{u}|^{2} \vec{n} \cdot \vec{e}_{z} \mathrm{~d} S-\rho \int_{V}(\vec{u} \times \vec{\omega}) \frac{\cdot \nabla \phi_{z}}{U_{\infty}} \mathrm{d} V+\mu \int_{S}(\vec{\omega} \times \vec{n}) \cdot\left(\frac{\nabla \phi_{z}}{U_{\infty}}+\vec{e}_{z}\right) \mathrm{d} S,
\end{aligned}
$$

where $\vec{u}$ is the velocity of the flow, $\vec{\omega}$ is the vorticity, $\mu$ is the dynamic viscosity of the fluid, $S$ the surface of the airfoil, $V$ the fluid domain, $\vec{n}$ the unitary vector normal to the surface of the airfoil, pointing towards the fluid, and $\vec{e}_{x}$ and $\vec{e}_{z}$ are the unitary vectors in the $x$ and $z$ directions, respectively. The auxiliary potentials $\phi_{x}$ and $\phi_{z}$ that appear in equation (2) depend only on the geometry of the airfoil and on the directions in which they are computed. For details of the calculations of these potentials, the reader is referred to the Appendix $B$.

Following Chang [1992], we group the terms of equation (2) to identify three different contributions to the aerodynamic forces,

$$
\vec{F}=\vec{F}^{m}+\vec{F}^{v}+\vec{F}^{s} .
$$

The first two terms of the right hand side in equation (2) are the contribution due to the motion of the body, $\vec{F}^{m}$. The contribution of the vorticity within the flow, $\vec{F}^{v}$, is given by the third term. Finally, the surface vorticity contribution, $\vec{F}^{s}$, is the last term of equation (2).

The decomposition described in equation (2) presents some advantages with respect to other algorithms found in the literature. First, the contribution of the body motion is calculated with surface integrals which only involve the velocity of the flow and the auxiliary potential functions, both on the surface of the airfoil. Hence, the contribution for $\vec{F}^{m}$ is prescribed by the geometry and the kinematics of the airfoil, and can be computed a priori (see section 4). Second, the only time derivative of the fluid velocity in equation (2) appears in a surface integral, so that $\frac{\partial \vec{u}}{\partial t}$ can be evaluated from the kinematics of the airfoil. This means that this force decomposition algorithm can be applied to isolated snapshots of the velocity field, e.g. obtained from particle image velocimetry measurements. Finally, the integrand of the contribution of the vorticity within the flow can be interpreted as a force density, allowing a direct evaluation of how specific vortices within the flow contribute to the total aerodynamic force.

The aerodynamic forces in equation (2) can be made dimensionless using the density $\rho$, the free stream velocity $U_{\infty}$ and the chord $c$, resulting in the non-dimensional coefficients of thrust $\left(c_{t}\right)$ and

\footnotetext{
${ }^{1}$ Note that there is some ambiguity in the literature about the definition of the reduced frequency. Some authors define it as $k=2 \pi f c / U_{\infty}$, while others as $k=\pi f c / U_{\infty}$. Here we have chosen the former.
} 


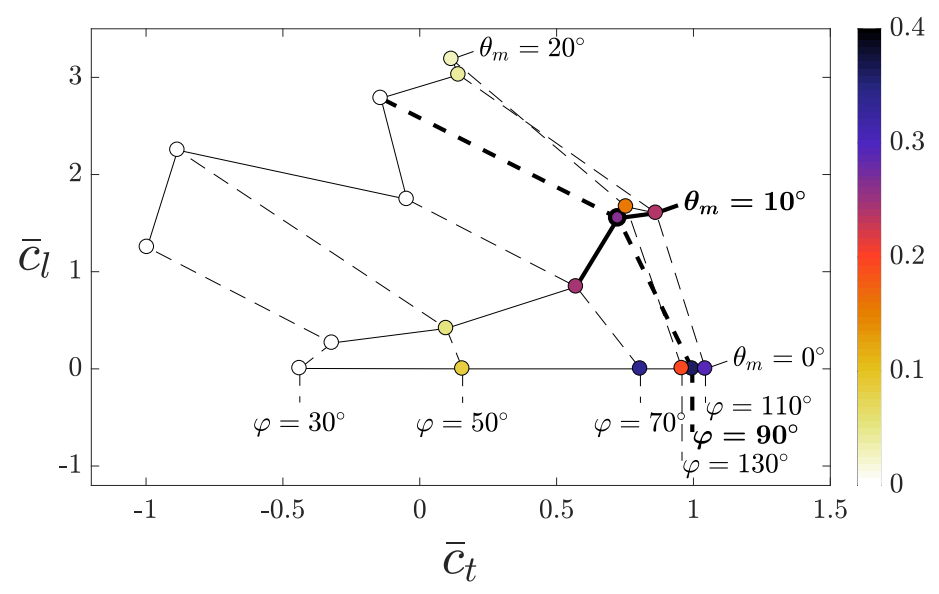

Figure 2: $\bar{c}_{l}$ vs. $\bar{c}_{t}$ for all cases in the database. The color corresponds to the propulsive efficiency, $\eta$. Solid (dashed) lines connect cases with constant mean pitch value $\theta_{m}$ (phase shift $\varphi$ ).

lift $\left(c_{l}\right)$

$$
c_{t}=\frac{-2 F_{x}}{\rho U_{\infty}^{2} c}, \quad c_{l}=\frac{2 F_{z}}{\rho U_{\infty}^{2} c} .
$$

Analogous to equation (3), the total non-dimensional force coefficients are split into

$$
\begin{aligned}
& c_{t}=c_{t}^{m}+c_{t}^{v}+c_{t}^{s}, \\
& c_{l}=c_{l}^{m}+c_{l}^{v}+c_{l}^{s} .
\end{aligned}
$$

Furthermore, we define the spatial density of thrust $\delta_{t}$ and lift $\delta_{l}$ as the integrand of the contribution of the vorticity within the flow to the total aerodynamic force in equation (2). After nondimensionalization, the definitions of $\delta_{t}$ and $\delta_{l}$ read

$$
\begin{aligned}
\delta_{t} & =\frac{2(\vec{u} \times \vec{\omega}) \cdot \nabla \phi_{x}}{U_{\infty}^{3} / c}, \\
\delta_{l} & =\frac{-2(\vec{u} \times \vec{\omega}) \cdot \nabla \phi_{z}}{U_{\infty}^{3} / c} .
\end{aligned}
$$

Also, to support the following discussions we define the average of any time dependent variable $g(t)$, as

$$
\bar{g}=\frac{1}{T_{a v}} \int_{0}^{T_{a v}} g(t) \mathrm{d} t,
$$

where $T_{a v}$ is the time span for the averaging process. Finally, the root mean square (rms) of $g(t)$ is defined as

$$
g^{\prime}=\sqrt{\frac{1}{T_{a v}} \int_{0}^{T_{a v}}\left(g(t)^{2}-\bar{g}^{2}\right) \mathrm{d} t .}
$$

\section{Aerodynamic forces}

Table 1 shows the averaged and rms values of thrust and lift coefficients for the 18 cases described in section 2, together with the propulsive efficiency

$$
\eta=\frac{T_{a v} \bar{F}_{x} U_{\infty}}{\int_{0}^{T_{a v}}\left(F_{z} \dot{h}+M_{y, c / 4} \dot{\theta}\right) \mathrm{d} t},
$$




\begin{tabular}{lrrrrrrrrc} 
Case & $\theta_{m}\left({ }^{\circ}\right)$ & $\varphi\left(^{\circ}\right)$ & $T_{a v} / T$ & Periodicity & \multicolumn{1}{c}{$\overline{c_{t}}$} & $c_{t}{ }^{\prime}$ & $\overline{c_{l}}$ & $c_{l}{ }^{\prime}$ & $\eta$ \\
\hline $\mathrm{A} 030$ & 0 & 30 & 10 & $\mathrm{~A}$ & -0.4382 & 0.6144 & 0.0041 & 3.3181 & 0 \\
$\mathrm{~A} 050$ & 0 & 50 & 1 & $\mathrm{P}$ & 0.1566 & 0.7184 & 0.0000 & 2.6276 & 0.0834 \\
$\mathrm{~A} 070$ & 0 & 70 & 1 & $\mathrm{P}$ & 0.8062 & 0.7550 & 0.0000 & 2.4396 & 0.3374 \\
$\mathrm{~A} 090$ & 0 & 90 & 1 & $\mathrm{P}$ & 0.9957 & 0.9751 & 0.0000 & 2.7164 & 0.3644 \\
$\mathrm{~A} 110$ & 0 & 110 & 1 & $\mathrm{P}$ & 1.0439 & 1.2878 & 0.0000 & 3.7486 & 0.2933 \\
$\mathrm{~A} 130$ & 0 & 130 & 1 & $\mathrm{P}$ & 0.9582 & 1.4988 & 0.0046 & 5.2013 & 0.1937 \\
$\mathrm{~B} 030$ & 10 & 30 & 5 & $\mathrm{~A}$ & -0.3202 & 0.7715 & 0.2662 & 3.0791 & 0 \\
$\mathrm{~B} 050$ & 10 & 50 & 1 & $\mathrm{P}$ & 0.0972 & 0.7235 & 0.4163 & 2.6734 & 0.0519 \\
$\mathrm{~B} 070$ & 10 & 70 & 1 & $\mathrm{P}$ & 0.5787 & 0.7017 & 0.8312 & 2.7040 & 0.2486 \\
$\mathrm{~B} 090$ & 10 & 90 & 1 & $\mathrm{P}$ & 0.7245 & 0.9224 & 1.5507 & 2.7743 & 0.2620 \\
$\mathrm{~B} 110$ & 10 & 110 & 1 & $\mathrm{P}$ & 0.8635 & 1.2008 & 1.6032 & 3.7622 & 0.2348 \\
$\mathrm{~B} 130$ & 10 & 130 & 1 & $\mathrm{P}$ & 0.7547 & 1.4351 & 1.6678 & 5.0979 & 0.1539 \\
$\mathrm{C} 030$ & 20 & 30 & 10 & $\mathrm{~A}$ & -0.9957 & 1.1545 & 1.2519 & 3.1465 & 0 \\
$\mathrm{C} 050$ & 20 & 50 & 1 & $\mathrm{P}$ & -0.8843 & 0.7999 & 2.2511 & 2.3009 & 0 \\
$\mathrm{C} 070$ & 20 & 70 & 15 & $\mathrm{~A}$ & -0.0468 & 0.5790 & 1.7455 & 2.7850 & 0 \\
$\mathrm{C} 090$ & 20 & 90 & 2 & $\mathrm{D}$ & -0.1419 & 0.9511 & 2.7850 & 2.9327 & 0 \\
$\mathrm{C} 110$ & 20 & 110 & 1 & $\mathrm{P}$ & 0.1425 & 1.0816 & 3.0268 & 3.7850 & 0.0392 \\
$\mathrm{C} 130$ & 20 & 130 & 2 & $\mathrm{D}$ & 0.1132 & 1.2640 & 3.1992 & 4.8421 & 0.0242
\end{tabular}

Table 1: Motion parameters and integrated values of non-dimensional force coefficients of thrust and lift of all the cases. The periodicity of the flow is indicated with $\mathrm{P}$ for periodic, $\mathrm{D}$ for periodic with period $2 T$ and $\mathrm{A}$ for aperiodic.

where $M_{y, c / 4}$ is the pitching moment about the quarter of the chord (coincident with the hinge point). Note that the propulsive efficiency is set to zero for cases with net drag, avoiding meaningless negative values. The cases are identified by a letter (A, B or C) related to the value of $\theta_{m}\left(0^{\circ}, 10^{\circ}\right.$ or $20^{\circ}$, respectively), followed by three digits that correspond to the value of $\varphi$. Table 1 also shows that most of the cases present the same periodicity in the flow and forces as in the motion, with period $T U_{\infty} / c=4.44$. However, there are two cases with a doubling period phenomena and four cases that are aperiodic. Therefore, the time span for the averaging $T_{a v}$ for the mean and rms coefficients of each case has been selected accordingly, as seen in the fourth column in table1. The appearance of aperiodic behavior has been previously observed by other authors, for example by Lewin and Haj-Hariri [2003] in pure heaving cases.

A graphical representation of the data in table 1 is provided in figure 2, where each case is represented by a point in the $\bar{c}_{t}, \bar{c}_{l}$ phase space. It can be seen that when the phase shift is fixed, an increase of the mean pitch value results in an increase of lift and a reduction of thrust (dashed lines in figure 2). When the mean pitch value is fixed (solid lines in figure 2), $\bar{c}_{t}$ and $\bar{c}_{l}$ tend to increase with $\varphi$. For moderate mean pitch angles, maximum propulsive efficiency is achieved for $\varphi \approx 90^{\circ}$, while maximum force coefficients are obtained for slightly larger phase shifts, $\varphi \approx 110^{\circ}$, consistent with the results of Anderson et al. 1998. More specifically, for a mean pitch value $\theta_{m}$ equal to $0^{\circ}$ and $10^{\circ}$, the maximum thrust is obtained for a phase shift $\varphi=110^{\circ}$. The highest propulsive efficiency is $36 \%$, obtained for $\theta_{m}=0^{\circ}$ and a phase shift $\varphi=90^{\circ}$. Also, cases with $\theta_{m}=10^{\circ}$ and $\varphi=90-110^{\circ}$ yield relatively high $\overline{c_{t}}$ and $\overline{c_{l}}$, with propulsive efficiencies higher than $20 \%$. Finally, note that for $\theta_{m}=20^{\circ}$ the propulsive efficiencies are considerably lower, with higher lift coefficients and a tendency to lose periodicity.

We select case B090 as a reference case to perform a more detailed analysis. This choice is motivated by the fact that B090 provides both thrust and lift with a relatively high propulsive efficiency, $\eta=26 \%$, so it is interesting in terms of aerodynamic performance. Also, by varying the phase shift or the mean 

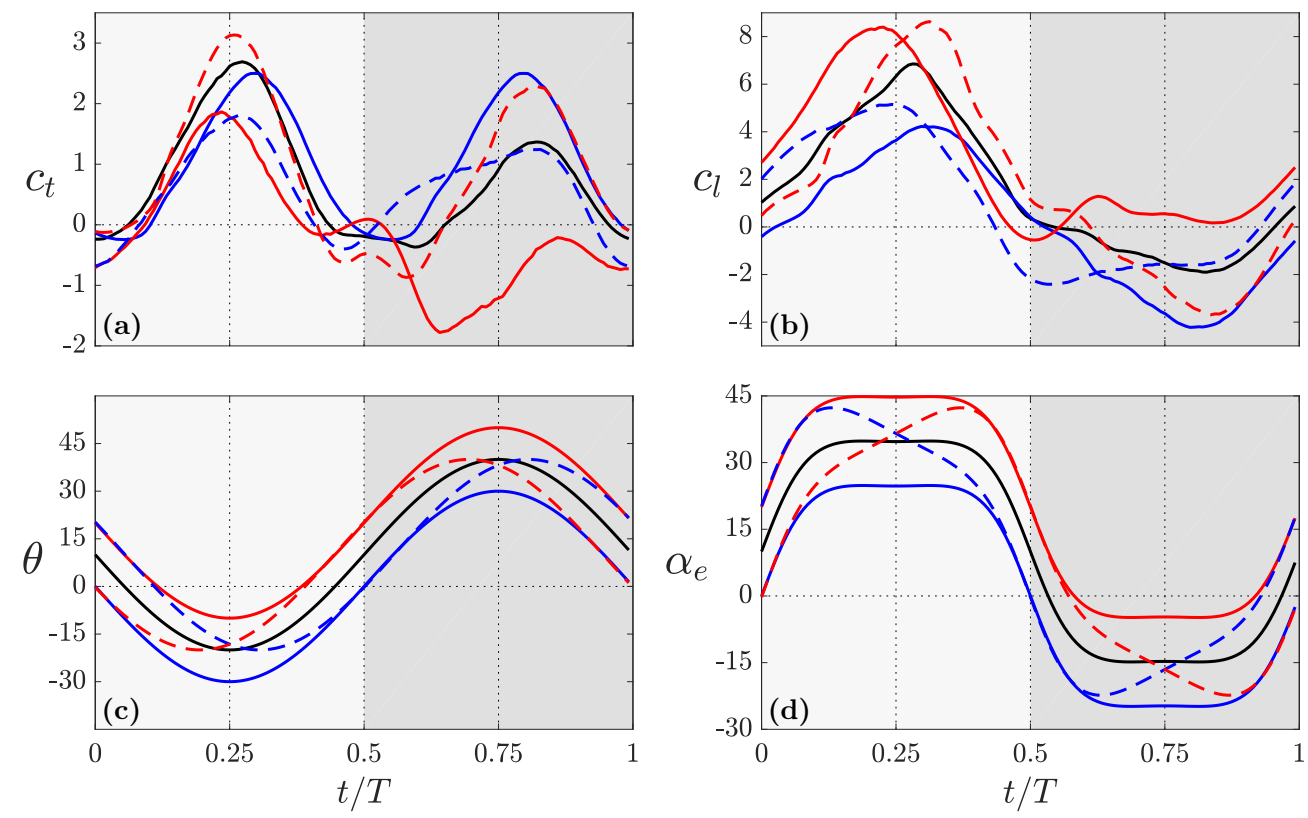

Figure 3: a) Thrust and b) lift coefficient of the selected cases with respect to the reference case B090 ( - ). c) Pitch angle of the airfoil and d) effective angle of attack (in degrees). Cases where $\theta_{m}$ is modified are represented with solid lines, case A090 ( - ) and case $\mathrm{C} 090(-)$, and cases where $\varphi$ is modified are represented with dashed lines, case B070 (-- ) and case B110 (--- ). The downstroke (upstroke) is indicated by a light (dark) grey background.

pitch value of case B090, a subset of cases from the database can defined, which allows the analysis of the influence of the motion parameters on the aerodynamic forces. This subset of cases is represented in figure 2 with thicker solid and dashed lines, and it includes cases A090, C090, B070 and B110.

In the reference case B090, the net thrust and lift $\left(\bar{c}_{t}=0.72, \bar{c}_{l}=1.55\right)$ are of the same order of magnitude as the rms of the force fluctuations $\left(c_{t}^{\prime}=0.92, c_{l}^{\prime}=2.77\right)$. This reflects that the oscillatory component of the force is as important as its mean value. For case B090, figures $3 \mathrm{a}$ and b show the time evolution of $c_{t}$ and $c_{l}$, respectively, during one period. Two peaks of thrust (figure 3 a) are generated during the period, one in the downstroke and one in the upstroke, with a larger magnitude in the former. Regarding the lift (figure 3 $\mathrm{p}$ ), the large lift generated in the downstroke is partially counteracted by the negative lift produced in the upstroke.

The influence of the mean pitch angle is analyzed in detail comparing cases A090, B090 and C090, which have a constant phase shift $\varphi$ of $90^{\circ}$ and a mean pitch value $\theta_{m}$ of $0^{\circ}, 10^{\circ}$ and $20^{\circ}$, respectively. If the mean pitch value $\theta_{m}$ is set to zero (case A090), the performance of the airfoil is improved with respect to case B090 $\left(\theta_{m}=10^{\circ}\right)$ in terms of net thrust, but at the cost of producing zero net lift. The $\bar{c}_{t}$ generated by case A090 is increased by $37.5 \%$ with respect to B090 and the propulsive efficiency by $40 \%$. Conversely, if the mean pitch value $\theta_{m}$ is increased to $20^{\circ}$ (case C090), a larger lift is generated $\left(\bar{c}_{l}=2.8\right)$, at the cost of producing a small net drag $\left(\bar{c}_{t}=-0.15\right)$. Concerning the variation of the force during the period, rms values of lift and thrust are comparable to mean values, as in the reference case. Moreover, $c_{t}^{\prime}$ and $c_{l}^{\prime}$ are roughly insensitive to variations of the mean pitch value, with $c_{t}^{\prime} \approx 0.9$ and $c_{l}^{\prime} \approx 2.8$ for cases A090, B090 and C090. In terms of the instantaneous forces, figure 3 a shows that, during the downstroke, the thrust generated in case A090 $\left(\theta_{m}=0^{\circ}\right)$ is similar to the thrust generated in case B090 $\left(\theta_{m}=10^{\circ}\right)$. However, during the upstroke the thrust generated is notably larger in case A090 compared to case B090. Conversely, case C090 $\left(\theta_{m}=20^{\circ}\right)$ generates less thrust during the whole period, with negative values of $c_{t}(t)$ during the upstroke, resulting in net drag. The 

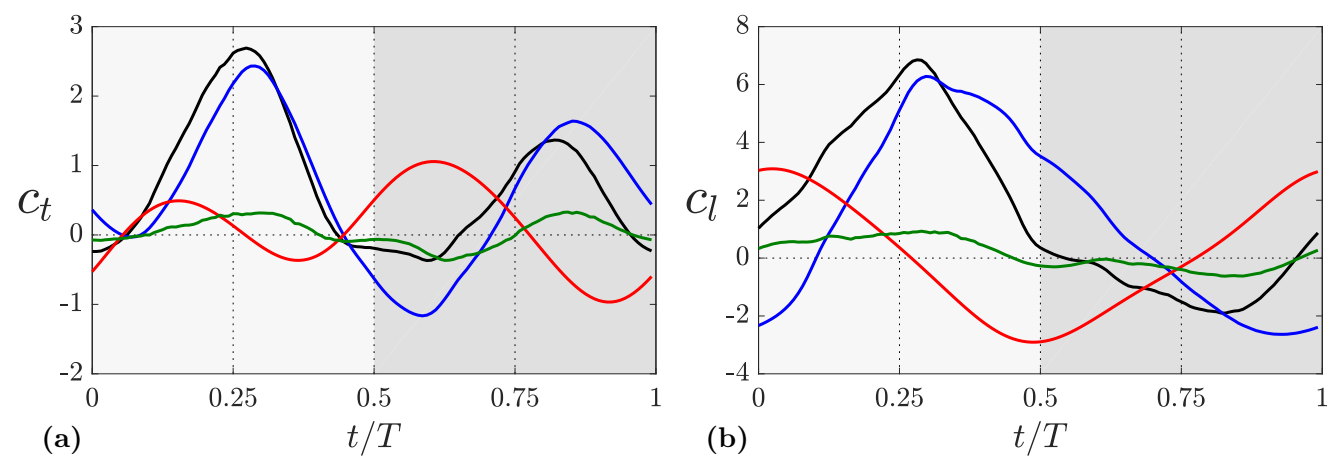

Figure 4: a) Thrust and b) lift coefficient of case B090. Curves represented correspond to the total aerodynamic force $\left(c_{l}, c_{t}\right.$ : - $)$ and contributions from body motion $\left(c_{l}^{m}, c_{t}^{m}:-\right)$, vorticity within the flow $\left(c_{l}^{v}, c_{t}^{v}\right.$ : - $)$ and surface vorticity $\left(c_{l}^{s}, c_{t}^{s}\right.$ : - $)$. The downstroke (upstroke) is indicated by a light (dark) grey background.

behavior of the instantaneous lift coefficient (figure $3 \mathrm{~b}$ ) is roughly the opposite, increasing with $\theta_{m}$ during the whole period, except in the transition from downstroke to upstroke. This transition is marked by a drop in $c_{l}$ associated to the detachment of the LEV, which occurs earlier for the cases with higher $\theta_{m}$. Overall, the variation of $c_{l}$ and $c_{t}$ with $\theta_{m}$ is consistent with an increase of the total force as $\theta_{m}$ increases, coupled with a change of the orientation of that force, which is tilted backwards as $\theta_{m}$ increases.

The effect of the phase shift on the aerodynamic forces is less intuitive. This effect is analyzed comparing cases B070, B090 and B110, which have a constant mean pitch value $\theta_{m}$ of $10^{\circ}$ and a phase shift $\varphi$ of $70^{\circ}, 90^{\circ}$ and $110^{\circ}$, respectively. A lag in the pitching motion $\left(\varphi<90^{\circ}\right.$, as in case B070) results in lower mean forces, specially for the mean lift coefficient. On the other hand, an advance of the pitching motion $\left(\varphi>90^{\circ}\right.$, as in case B110) results in higher net thrust and lift. Besides the change on the mean values, the main effect of the phase shift on the instantaneous force coefficients is an increase of the amplitude of the force perturbations with $\varphi$. This can be observed both in figures $3 \mathrm{a}$ and $\mathrm{b}$, as well as in the $c_{t}^{\prime}$ and $c_{l}^{\prime}$ reported in table 1 . Finally, it is clear from figure $3 a$ that $\varphi$ also modifies the time at which the lift coefficients are maximum. This effect on the lift can be partly explained by the evolution of the effective angle of attack $\alpha_{e}=\theta-\operatorname{atan}\left(\dot{h} / U_{\infty}\right)$, shown in figure $3 \mathrm{~d}$. For $\varphi>90^{\circ}(\mathrm{B} 110)$, the peaks of $\alpha_{e}$ are delayed in time with respect to B090. As a consequence, the peaks of $c_{l}$ occur later for B110 than for B090. On the other hand, for $\varphi<90^{\circ}$ (B070), the peaks of $\alpha_{e}$ and $c_{l}$ are advanced with respect to B090. Note that a similar trend is not apparent in the times where $c_{t}$ is maximum, which are fairly independent of the phase shift. The reason for this is the importance of the pitch angle in the orientation of the resulting aerodynamic force: if we assume that the aerodynamic forces are mostly perpendicular to the airfoil (an assumption that will be justified later), then the variation in the pitch angle during the upstroke or downstroke between cases B070, B090 and B110 results in a small effect for the lift (which is multiplied by $\cos \theta$ ), but a larger effect on the thrust (which is multiplied by $\sin \theta$ ). Note that for these cases, the pitch angle, $\theta$, shown in figure 3 ; , is such that $\cos \theta \sim 1$ and $\sin \theta \sim \theta$. As a consequence, the lift is dominated by $\alpha_{e}$ while the thrust depends on both, $\alpha_{e}$ and $\theta$.

\section{Force decomposition and modelling of case B090}

Following the procedure described in section 2, we decompose the total aerodynamic force of case B090. Figure 4 shows the evolution of the total thrust and lift, together with the contributions from 
body motion, $\vec{F}^{m}$, vorticity within the flow, $\vec{F}^{v}$, and surface vorticity, $\vec{F}^{s}$, during one period of case B090. The main contribution to the total aerodynamic force corresponds to the vorticity within the flow, with peak values of the same order of magnitude as the total value of the force. Body motion has also an important role in the generation of force, producing peak values around half of the peak values of the total aerodynamic force. Finally, surface vorticity (viscous effects) is the least important contribution, with peak values approximately ten times smaller than the total force peak values. Therefore, in the following we will focus in the analysis of the contributions from body motion and the vorticity within the flow.

We start with the contribution of the body motion to the total force, $\vec{F}^{m}$, which is the force produced by the fluid to counteract the motion of the airfoil. This is easily observed in figure $4 \mathrm{~b}$ : when $\ddot{h}<0, c_{l}^{m}$ is a positive vertical force, and viceversa. The thrust (figure 4 a) is influenced by both the vertical acceleration and the projected area of the airfoil perpendicular to the streamwise direction. For the motion parameters of this case $\left(\theta_{m}=10^{\circ}, \varphi=90^{\circ}\right)$, the projected area during the downstroke is smaller than the projected area during the upstroke, resulting in higher peaks of $c_{t}^{m}$ in the latter.

From a physical point of view, $\vec{F}^{m}$ is similar to the added-mass term of the aerodynamic forces in unsteady potential flow. However, they are not exactly the same. This is shown here for a flat plate with $x_{p}=c / 2$. According to Sedov 1965, the added mass forces for this configuration are

$$
\begin{aligned}
& c_{t}^{a}=\frac{\pi}{4} \frac{\ddot{h}}{U_{\infty}^{2} / c} \sin (2 \theta)+\frac{\pi}{2} \frac{\dot{h}}{U_{\infty}} \frac{\dot{\theta}}{U_{\infty} / c} \cos ^{2}(\theta)-\frac{\pi}{4}\left(\frac{\dot{\theta}^{2}}{U_{\infty}^{2} / c^{2}} \cos \theta+\frac{\ddot{\theta}}{U_{\infty}^{2} / c^{2}} \sin \theta\right), \\
& c_{l}^{a}=-\frac{\pi}{2} \frac{\ddot{h}}{U_{\infty}^{2} / c} \cos ^{2} \theta+\frac{\pi}{4} \frac{\dot{h}}{U_{\infty}} \frac{\dot{\theta}}{U_{\infty} / c} \sin (2 \theta)+\frac{\pi}{4}\left(\frac{\ddot{\theta}}{U_{\infty}^{2} / c^{2}} \cos \theta-\frac{\dot{\theta}^{2}}{U_{\infty}^{2} / c^{2}} \sin \theta\right) .
\end{aligned}
$$

However, the corresponding expressions for the $c_{t}^{m}$ and $c_{l}^{m}$ are

$$
\begin{aligned}
c_{t}^{m} & =\frac{\pi}{4} \frac{\ddot{h}}{U_{\infty}^{2} / c} \sin (2 \theta), \\
c_{l}^{m} & =-\frac{\pi}{2} \frac{\ddot{h}}{U_{\infty}^{2} / c} \cos ^{2} \theta,
\end{aligned}
$$

where the analytical expressions for the auxiliary potentials of a flat plate are obtained from MartínAlcántara et al., 2015. Note that while the first term in equation (10) is the same appearing in equation (11), the former has some extra terms depending explicitly on $\dot{\theta}$ and $\ddot{\theta}$. Moreover, for periodic motions the mean value of the added mass coefficients are $\bar{c}_{l}^{a}=\bar{c}_{t}^{a}=0$, while the mean value of the body motion contributions are only zero if $\varphi=90^{\circ}$. This can be observed in figure 5, which shows $\bar{c}_{t}{ }^{m}$ and $\bar{c}_{l}{ }^{m}$ as a function of $\varphi$ for $\theta_{m}=0^{\circ}, 10^{\circ}$ and $20^{\circ}$. Interestingly, the behaviour of $\bar{c}_{t}{ }^{m}$ and $\bar{c}_{l}{ }^{m}$ in figure 5 is consistent with the trends shown in figure 2 , except for maybe the behavior for $\varphi \gtrsim 110^{\circ}$.

Finally, from the point of view of modelling, it is interesting to note that $\vec{F}^{m}$ can be computed a priori, since the velocity on the surface is known from the airfoil's kinematics. Note also that, as explained in appendix $B$, the auxiliary potentials can be computed on a reference frame fixed to the airfoil, and then rotated and translated appropriately to account for the motion of the airfoil. For reference, the auxiliary potentials for the NACA 0012 airfoil used in this work are provided in figure 17 in appendix B.

Now, we turn our attention to the contribution that the vorticity within the flow has on the total aerodynamic force of case B090 (blue lines in figure 44). It is clear that the (positive) peaks of total thrust and lift are dominated by the contribution of the vorticity within the flow. Also, the contribution of the vorticity within the flow is maximum when the vertical velocity of the airfoil $\dot{h}$ and the effective angle of attack $\alpha_{e}$ are maximum. Therefore, two peaks of positive thrust are observed in figure 4 , slightly lagged with respect to the middle of the downstroke $(t / T=0.25)$ and the middle 

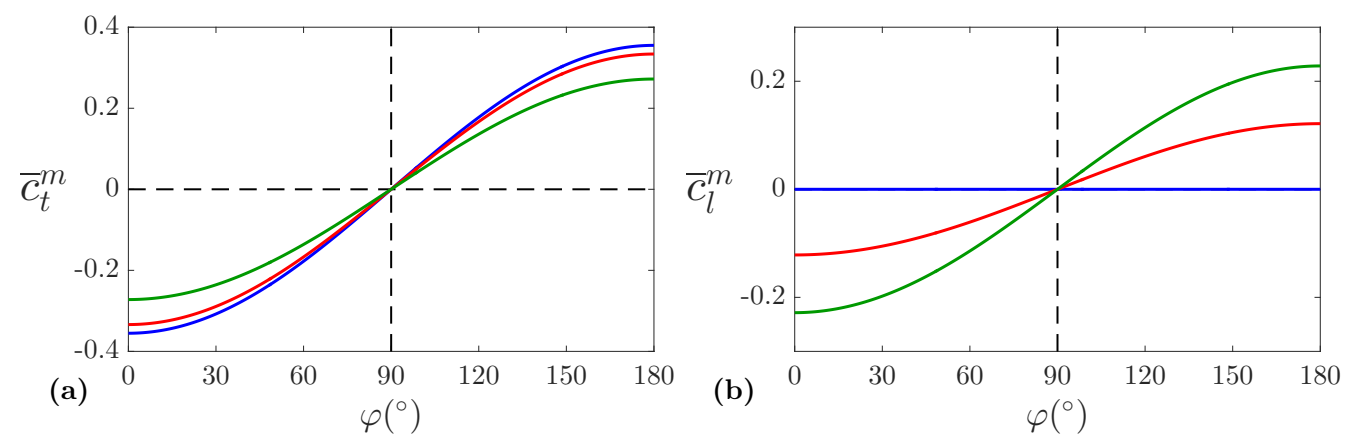

Figure 5: Mean a) thrust and b) lift coefficients from body motion contribution for a flat plate with respect to the phase shift $\varphi$. The values shown correspond to different values of mean pitch angle, $\theta_{m}=0^{\circ}(-), \theta_{m}=10^{\circ}(-)$ and $\theta_{m}=20^{\circ}(-)$.

of the upstroke $(t / T=0.75)$, respectively. Regarding the lift, the peak of the instantaneous force coefficients are also slightly lagged. The peak of force generated around the middle of the downstroke $(t / T=0.25)$ is positive and the one generated around the middle of the upstroke $(t / T=0.75)$ is negative. The asymmetry introduced by the mean pitch angle $\theta_{m}=10^{\circ}$ results in lower peak values in the upstroke compared to the downstroke, which is detrimental for the thrust, but favourable for the lift.

In order to obtain a better understanding, we continue the analysis of case B090 by comparing the spanwise vorticity, $\omega_{y}$, and the force density fields of the contribution of vorticity within the flow at four equispaced time instants during one period, shown in figure 6. Both thrust $\delta_{t}$ and lift $\delta_{l}$ densities (figures 6b, e, h, k and 6c, f, i, l, respectively) decay fast with the distance to the airfoil for any time instant, as previously observed by other authors [Chang, 1992, Martín-Alcántara et al., 2015]. This occurs because the force density is the projection of the Lamb's vector on the gradient of the auxiliary potentials $\phi_{x}$ and $\phi_{z}$, which decay quadratically with the distance to the airfoil Martín-Alcántara et al. 2015]. The evolution of the spanwise vorticity (figures 6a, $\mathrm{d}, \mathrm{g}$ and $\mathrm{j}$ ) shows how the LEV is created during the downstroke (figure $6 \mathrm{~d}$ ) and shed into the wake approximately in the transition from downstroke to upstroke (figure 6s). At that time, the contribution of the LEV to the thrust changes sign, while its contribution to the lift remains positive for longer times. After being shed (figure 6j), the LEV is advected into the wake (figures $6 \mathrm{a}, \mathrm{d}$ and $\mathrm{g}$ ), and its contribution to the aerodynamic forces becomes negligible. The small influence on the aerodynamic forces from the vortices in the wake is consistent with the results of Moriche et al. [2016], where 2D and 3D configurations of infinite aspect ratio wings were found to yield very similar lift and thrust.

It is possible to observe in figure 6 that the contribution of the LEV to the thrust and lift is partially positive and negative. This is an inherent property of any vortex, as indicated by Chang [1992]: the center of a vortex is characterized by a change of direction of the Lamb's vector while the gradient of the auxiliary potentials is locally smooth, so a line where the force density changes its sign must pass through the center of the vortex. Which part of the vortex (positive and negative contribution to the force) dominates, depends on the vorticity field and on the gradients of the auxiliary potentials, which are determined only by the geometry of the airfoil. This is an interesting fact, which could be used to guide an optimisation of the airfoil shape.

It is clear from the previous discussion that the contribution of the vorticity within the flow is the dominant contribution to the total aerodynamic force. Therefore it is of great interest to model this part of the force appropriately. In steady-state aerodynamics and in several reduced order models in the literature (e.g, Pesavento and Wang, 2004, Andersen et al., 2005, Taha et al., 2014), it is common to use the Kutta-Joukowsky theorem to model the aerodynamic force due to the vorticity within the 


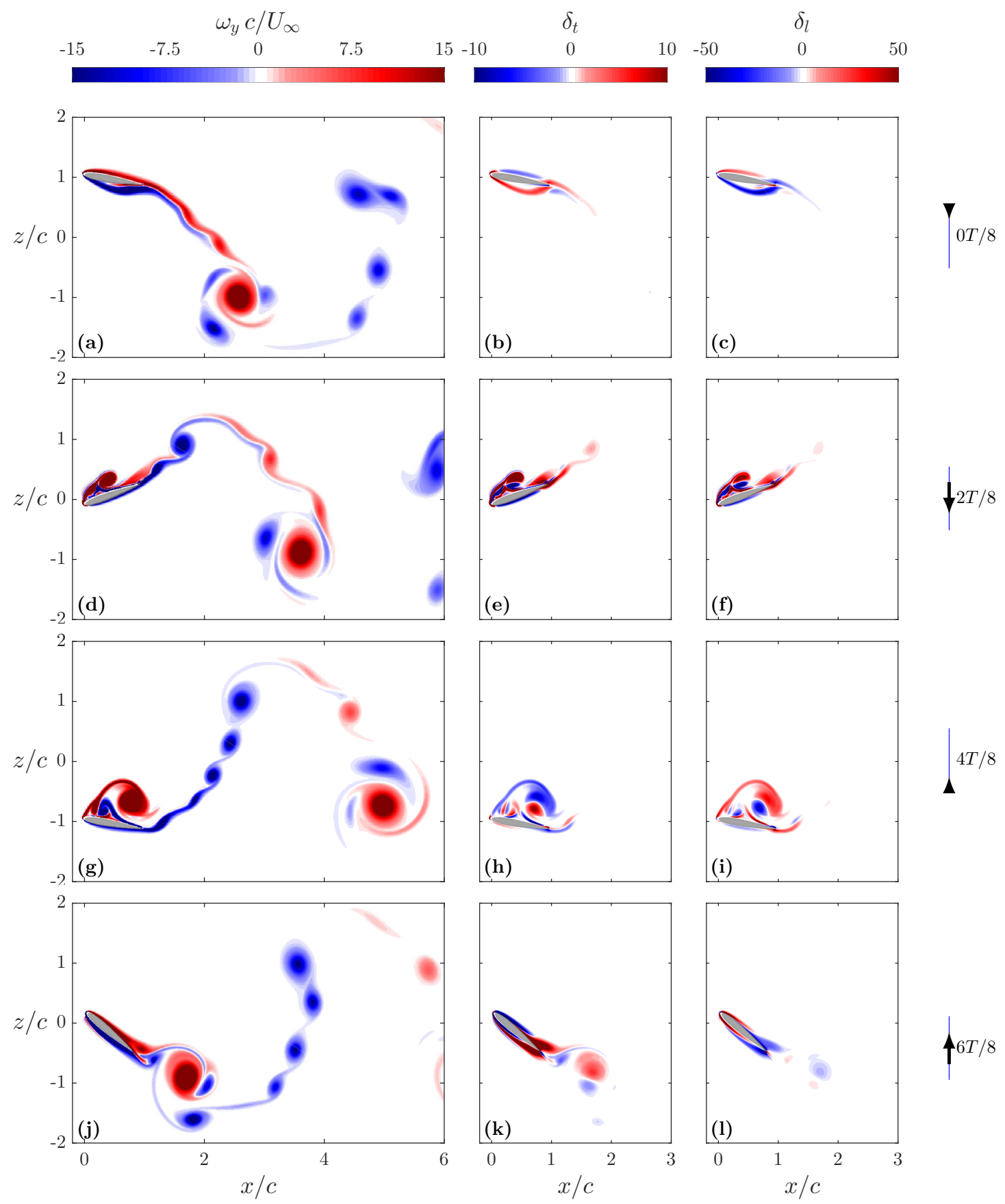

Figure 6: Contours of spanwise vorticity $\omega_{y}\left(\mathrm{a}, \mathrm{d}, \mathrm{g}\right.$ and $\mathrm{j}$ ), thrust density $\delta_{t}(\mathrm{~b}, \mathrm{e}, \mathrm{h}$ and $\mathrm{k}$ ) and lift density $\delta_{l}$ (c, d, i and l) of case B090 at four time instants. See the corresponding animations in the additional material. 


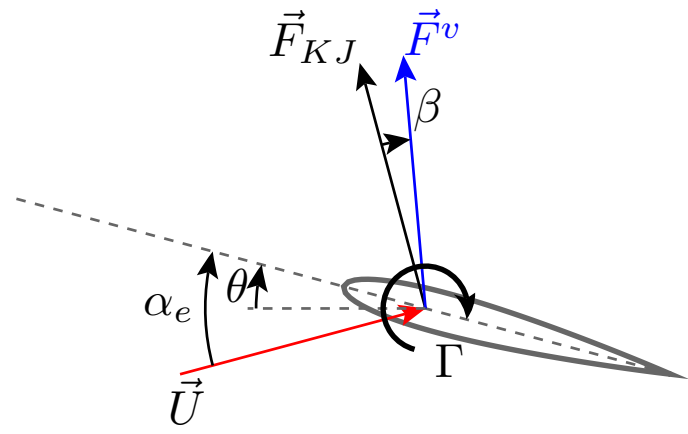

Figure 7: Sketch of the deviation angle $\beta$ and effective angle of attack $\alpha_{e}$.
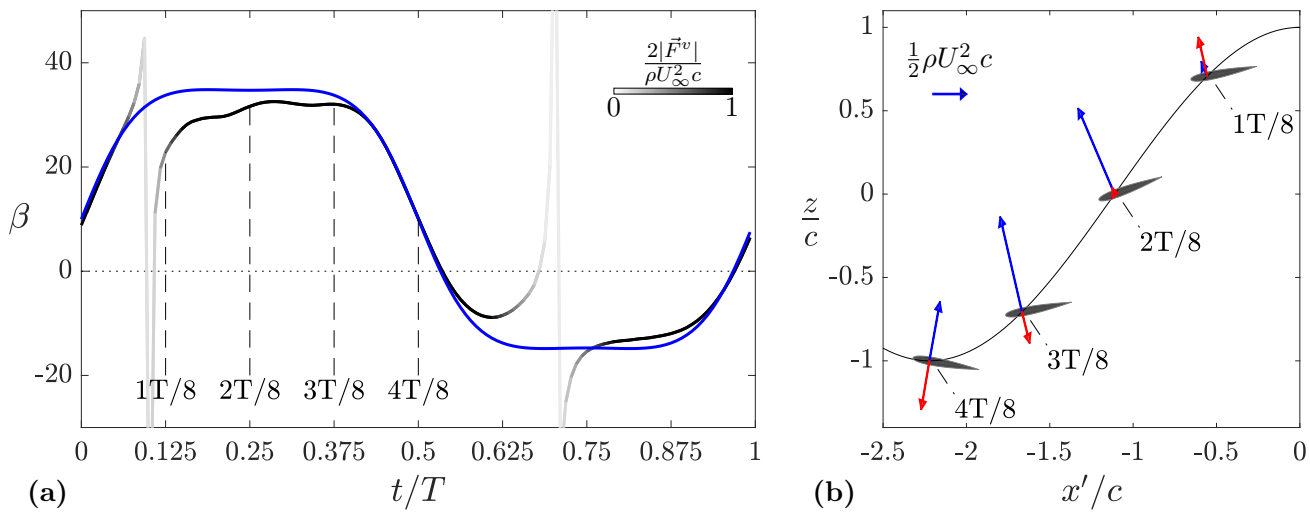

Figure 8: a) Evolution of angle $\beta$ (in degrees) of case B090 during one period. The curves represented are the angle $\beta$ in gray scale to indicate the modulus of the contribution of vorticity within the flow and the effective angle of attack $(-)$. b) Vectors of $\vec{F}^{v}(-)$ and $\vec{F}^{m}(-)$ at four time instants during the downstroke of case B090.

flow,

$$
\vec{F}_{K J}=\rho\left(\vec{U} \times \Gamma \vec{e}_{y}\right)
$$

where the subscript $K J$ denotes Kutta-Joukowsky estimation, $\Gamma$ is the circulation around the airfoil, $\vec{U}$ is the effective velocity seen by the airfoil and $\vec{e}_{y}$ is the unitary vector in the $y$ direction. The force estimated by Kutta-Joukowsky theorem is perpendicular to the incoming effective velocity, which can be estimated for a heaving and pitching airfoil as $\vec{U}=U_{\infty} \vec{e}_{x}-\dot{h}_{z}$, as described in Pesavento and Wang [2004], Andersen et al. [2005] and Taha et al. [2014].

In order to compare $F_{K J}$ and $\vec{F}^{v}$, we define the angle $\beta$ formed by these two vectors, as sketched in figure 7. The evolution of this angle $\beta$ for case B090 during one period is shown in figure 8 8 , where the curve representing $\beta$ is coloured with a grey scale proportional to $\left|\vec{F}^{v}\right|$. This is done to avoid confusion when the force tends to zero, situation where the angle $\beta$ is ill-defined. The evolution of the effective angle of attack $\alpha_{e}$ has been also included in the figure, and it can be clearly seen that the angle $\beta$ tends to $\alpha_{e}$, except for specific time instants where the force is small. This means that $\vec{F}^{v}$ is not oriented in the direction suggested by the Kutta-Joukowsky theorem (namely, normal to the incoming effective velocity), but $\vec{F}^{v}$ is approximately perpendicular to the chord of the airfoil. This observation is consistent with the empirical model of Dickinson et al. [1999, which results in aerodynamic forces essentially normal to the wing. The tendency to a chord-normal orientation of $\vec{F}^{v}$ is observed for all the periodic cases of our database. 


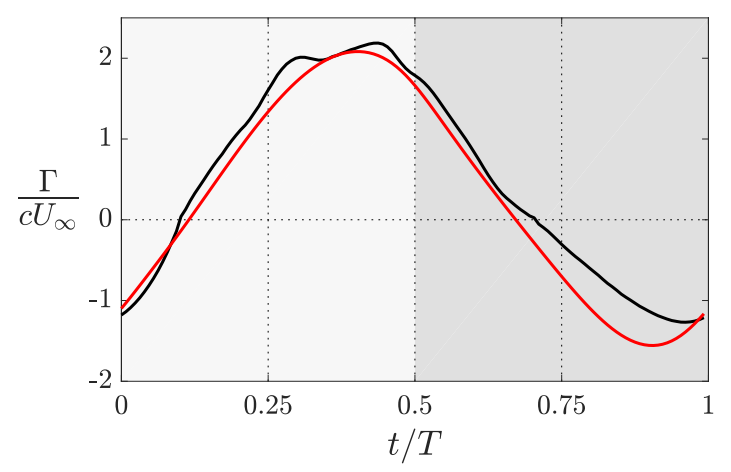

Figure 9: Circulation of the airfoil for case B090. The curves represented correspond to the values obtained from the DNS ( - ) and the model best fit ( - ) with $G_{T}=1.65$ and $G_{R}=3.73$. The downstroke (upstroke) is indicated by a light (dark) grey background.

Figure 8b shows a sketch of $\vec{F}^{v}$ and $\vec{F}^{m}$ acting on the airfoil at four equispaced time instants. In the figure the airfoil trajectory is represented as seen by an observer travelling with the free stream, so the horizontal coordinate is defined as $x^{\prime}=x-U_{\infty} t$. Note that the approximate chord-normal orientation of $\vec{F}^{v}$ occurs when the forces are large enough, which is consistent with a well developed LEV moving the suction peak from the leading edge of the airfoil to the upper surface of the airfoil. Also, figure 8 a shows that there is a small deviation of $\vec{F}^{v}$ with respect to the normal, of the order of $\pm 5^{\circ}$, which could result in a small component of $\vec{F}^{v}$ in the direction of the chord. Interestingly, $\vec{F}^{m}$ is also approximately perpendicular to the airfoil chord. Indeed, equations (11) show that this is strictly true for a flat plate pitching around $x_{p}=c / 2$.

From the point of view of modelling, the results of figure 8 suggest that $\vec{F}^{v}$ is a force roughly perpendicular to the airfoil chord. In order to estimate its modulus, we follow previous works and use the Kutta-Joukowsky theorem

$$
\left|\vec{F}^{v}\right|=\rho \Gamma|\vec{U}|,
$$

estimating the circulation of the airfoil as in Pesavento and Wang [2004],

$$
\Gamma=\frac{1}{2} G_{T} c|\vec{U}| \sin \left(2 \alpha_{e}\right)+\frac{1}{2} G_{R} c^{2} \dot{\theta} .
$$

In this expression, $G_{T}$ and $G_{R}$ are the two free parameters of the model. Note that (14) includes information of the amplitudes and frequency of the pitching and heaving motion of the airfoil in $\vec{U}, \alpha_{e}$ and $\theta$.

Figure 9 shows the comparison of the circulation obtained from the DNS (using the computed $\vec{F}^{v}$ and equation 13) and the circulation obtained from (14). For the latter, the constants $G_{T}=1.65$ and $G_{R}=3.75$ are obtained from a least square fit to the circulation from the DNS. It can be seen that the agreement is very good, except for the last part of the upstroke $(t / T>0.75)$, where the model slightly overpredicts the circulation.

Based on the observations made in this section, we propose modelling $\vec{F}^{v}$ as a force oriented normal to the chord, whose modulus is calculated with equations (13) and (14). Figure 10 shows the comparison between this chord-normal model, the Kutta-Joukowsky prediction (i.e., same modulus but perpendicular to $\vec{U}$ ) and the results of the DNS. The results show that peak values of thrust (figure 10a) are well predicted by the chord-normal force, but overestimated by Kutta-Joukowsky prediction. Regarding the lift, figure $10 \mathrm{~b}$ shows that $c_{l}^{v}$ is well reproduced by the chord-normal estimation, with a visible deviation corresponding to the already mentioned misrepresentation of $\Gamma$ near the end of the upstroke. 


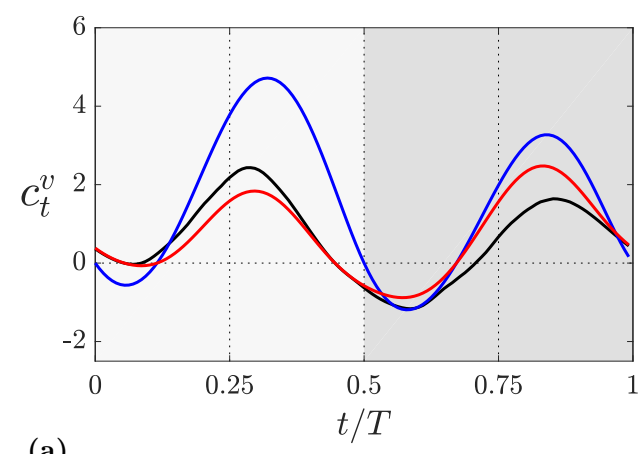

(a)

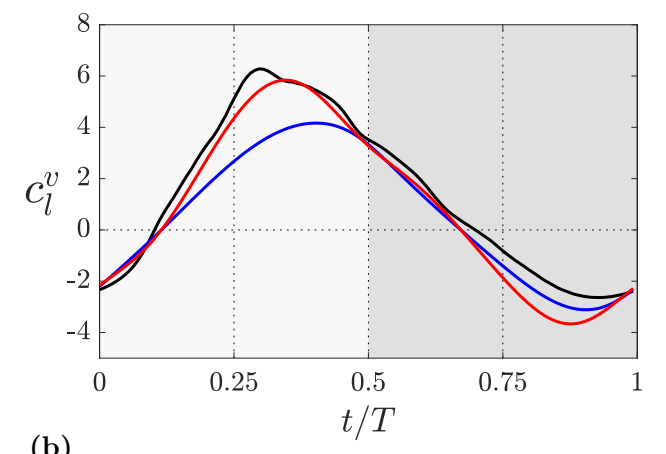

(b)

Figure 10: Evolution of a) $c_{t}^{v}$ and b) $c_{l}^{v}$ of case B090 during one period. The curves represented correspond to the values obtained from the DNS ( - ), the Kutta-Joukowsky estimation $(-)$ and the chord-normal estimation ( - ). The circulation in both estimations is given by the model from Pesavento and Wang with $G_{T}=1.65$ and $G_{R}=3.73$. The downstroke (upstroke) is indicated by a light (dark) grey background.

\section{Effects of mean pitch angle and phase shift in the force decompo- sition and modelling}

In this section we extend the analysis performed in the previous section on case B090 to a subset of cases from the database. The objective of extending the analysis is to see how the different contributions to the total aerodynamic force are influenced by the motion parameters $\theta_{m}$ and $\varphi$. As before, we select the cases A090, C090, B070 and B110 and use the results of case B090 as a reference.

Figure 11 shows the contributions to the lift and thrust coefficients from the vorticity within the flow, body motion and surface vorticity for the selected cases. Overall, figure 11a and b show that increasing $\theta_{m}$ increases $c_{l}^{v}$ and reduces $c_{t}^{v}$. This is because increasing $\theta_{m}$ increases the effective angle of attack (figure 3.), increasing the modulus of $\vec{F}^{v}$, and tilting the resultant backwards. Hence, reducing thrust and increasing lift. Also, the LEV detaches from the airfoil earlier as $\theta_{m}$ increases, shifting the peaks in lift during the downstroke to earlier times (see figure 11b). As mentioned earlier, this has a small effect on the thrust, which is strongly influenced by the orientation of the airfoil. During the upstroke, increasing $\theta_{m}$ reduces the (negative) effective angle of attack. This results in lower forces, although better oriented for thrust. For C090, at the beginning of the upstroke the pitch angle is large enough so that the airfoil is still producing positive lift, and since it is tilted backwards, drag (see $\theta$ and $\alpha_{e}$ in figure $3 c$ and $\mathrm{d}$, respectively).

As with the total forces, the variation of phase shift produces a different effect, affecting mainly the amplitude of the peak values of $\vec{F}^{v}$. An advance of the pitching motion (case B110) increases the amplitude of the fluctuations of both thrust and lift, whereas lagging the pitching motion (case B070) results in a reduction of these amplitudes. There is also an effect of the phase shift in the position of the peaks of $c_{l}^{v}$ during the downstroke, although this effect is not appreciable in $c_{l}^{v}$ during the upstroke, nor in the peaks of $c_{t}^{v}$.

Figures 11. and $\mathrm{d}$ show the evolution of $c_{t}^{m}$ and $c_{l}^{m}$, respectively, for the selected cases. For the motion under study, the heaving acceleration $\ddot{h}(t)$ dominates the contribution of body motion to the lift, independently of the parameters $\theta_{m}$ and $\varphi$ (figure $11 \mathrm{~d}$ ). Concerning the thrust, both the heaving acceleration $\ddot{h}(t)$ and the projected area of the airfoil perpendicular to the streamwise direction influence $c_{t}^{m}$. Therefore, $c_{t}^{m}$ vanishes at points where the heaving acceleration $\ddot{h}(t)$ is zero $(t / T=0.25,0.75)$. At points where the heaving acceleration $\ddot{h}(t)$ is maximum $(t / T=0,0.5)$, the value of $c_{t}^{m}$ depends on the area of the airfoil projected perpendicular to the streamwise direction. This area is related to the value of the pitch angle $\theta$ (figure 3 ). At the beginning of the downstroke 

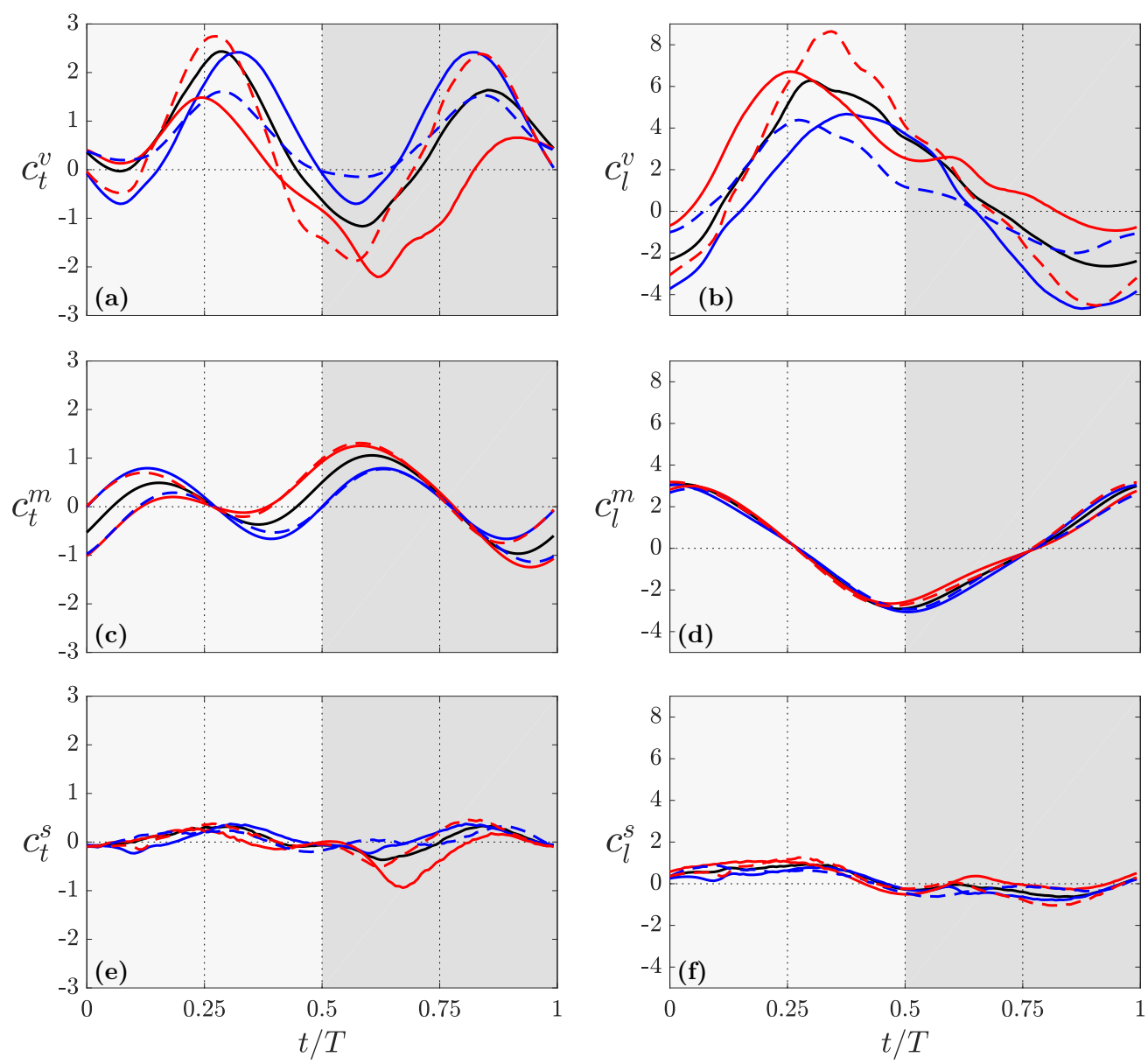

Figure 11: Contribution of a) vorticity within the flow, c) body motion and e) surface vorticity to the total aerodynamic thrust. The lift contributions are shown in b), d) and f), respectively. Four cases with different motion parameters are represented: case B090 ( - ), case A090 ( - ) , case C090 ( - ), case B070 (--- ) and case B110 (--- ). The downstroke (upstroke) is indicated by a light (dark) grey background. 


\begin{tabular}{crrrrcc} 
Case & $\theta_{m}$ & $\varphi$ & $G_{T}$ & $G_{R}$ & \multicolumn{2}{c}{$\left\|\Gamma_{D N S}-\Gamma\right\|_{2}$} \\
& & & & & Specific & Fixed \\
\hline B090 & $0.00^{\circ}$ & $90.00^{\circ}$ & 1.65 & 3.73 & 0.26 & 0.32 \\
A090 & $10.00^{\circ}$ & $90.00^{\circ}$ & 1.44 & 4.48 & 0.13 & 0.43 \\
C090 & $20.00^{\circ}$ & $90.00^{\circ}$ & 1.95 & 2.14 & 0.42 & 0.50 \\
B070 & $10.00^{\circ}$ & $70.00^{\circ}$ & 1.65 & 2.46 & 0.14 & 0.20 \\
B110 & $10.00^{\circ}$ & $110.00^{\circ}$ & 1.34 & 4.58 & 0.35 & 0.50
\end{tabular}

Table 2: Coefficients of Pesavento and Wang [2004] model for circulation (equation (14)) obtained from the best fit with the data from the DNS for the selected cases. The errors shown correspond to the circulation obtained with the coefficients obtained specifically for each case (next-to-last) column and fixed coefficients $G_{T}=1.85$ and $G_{R}=\pi$ (last column).

$(t / T=0)$, cases A090 and B110 have a pitch angle $\theta$ of $0^{\circ}$, so $c_{t}^{m}$ is roughly zero (figure 11). At this time instant, $c_{t}^{m}$ increases with the instantanous pitch angle, so case B090 generates more thrust than cases A090 and B110 and less than cases C090 and B070. The same analysis holds at the beginning of the upstroke $(t / T=0.5)$, except that, in this part of the period, cases with different phase shift (B070 and B110) interchange their role.

To conclude the analysis of the different contributions to the total aerodynamic force, figures 11 e and $\mathrm{f}$ show the evolution of the surface vorticity contribution to the total aerodynamic thrust and lift, respectively. It can be seen that the effects that surface vorticity has on the forces is small compared to the other contributions for both thrust and lift, except for a peak of viscous drag in the upstroke of case C090. A possible explanation is related to the fact that the effective angle of attack during the upstroke is close to zero, as shown in figure $3 \mathrm{~d}$, unlike the other cases considered. As a consequence, during the first half of the upstroke, the boundary layer in the lower surface is attached and thinner than in the other cases, with no appreciable LEV in the lower surface of the airfoil. This has been observed in visual inspection of the corresponding flow fields (not shown here). Hence, the skin friction in the lower surface of the airfoil is larger for case C090.

After describing the evolution of the different contributions to the total aerodynamic force, we proceed to check the capability of the chord-normal model proposed in the previous section to predict the contribution of the vorticity within the flow to the total aerodynamic force of the subset of cases A090, C090, B070 and B110. The first question is which values of $G_{T}$ and $G_{R}$ should be used in equation (14). In principle, one could obtain "specific" values for $G_{T}$ and $G_{R}$ for each case, repeating the same least square fitting process described in section 4 but for cases A090, C090, B070 and B110. These specific coefficients, as well as the corresponding L2 norm of the difference between the model and DNS circulation, are shown in table 2. It should be noted that, even if the variability of the coefficients with $\varphi$ and $\theta_{m}$ is not small (around $\pm 19 \%$ for $G_{T}$ and $\pm 35 \%$ for $G_{R}$ ), the assumption in Pesavento and Wang 2004 is that the coefficients $G_{T}$ and $G_{R}$ depend on the geometry, not on the kinematics. Indeed, the kinematic of the airfoil enters in equation (14) through $\alpha_{e}$ and $\theta$.

To check the validity of this assumption, we have also computed "fixed" values of these constants, choosing $G_{R}=\pi$ and fitting $G_{T}$ for all the periodic cases in table 1 using a least square method. The rationale for choosing $G_{R}=\pi$ comes from potential theory, which results in

$$
\Gamma_{R O T}=\pi c^{2} \dot{\theta}\left(\frac{3}{4}-\frac{x_{p}}{c}\right)
$$

for a thin airfoil in piching motion (this can be derived from the expressions appearing in section 6.2 of Fung, 2002). The resulting $G_{T}=1.85$, which is approximately the mean value of the "specific" values obtained for $G_{T}$, yields errors in the circulation $\Gamma$ of the same order of magnitude (see last column in table 2). Note also that from the point of view of modelling, "fixed" values of $G_{T}$ and $G_{R}$ are more interesting than "specific" values for these constants, since the former can be used to predict 

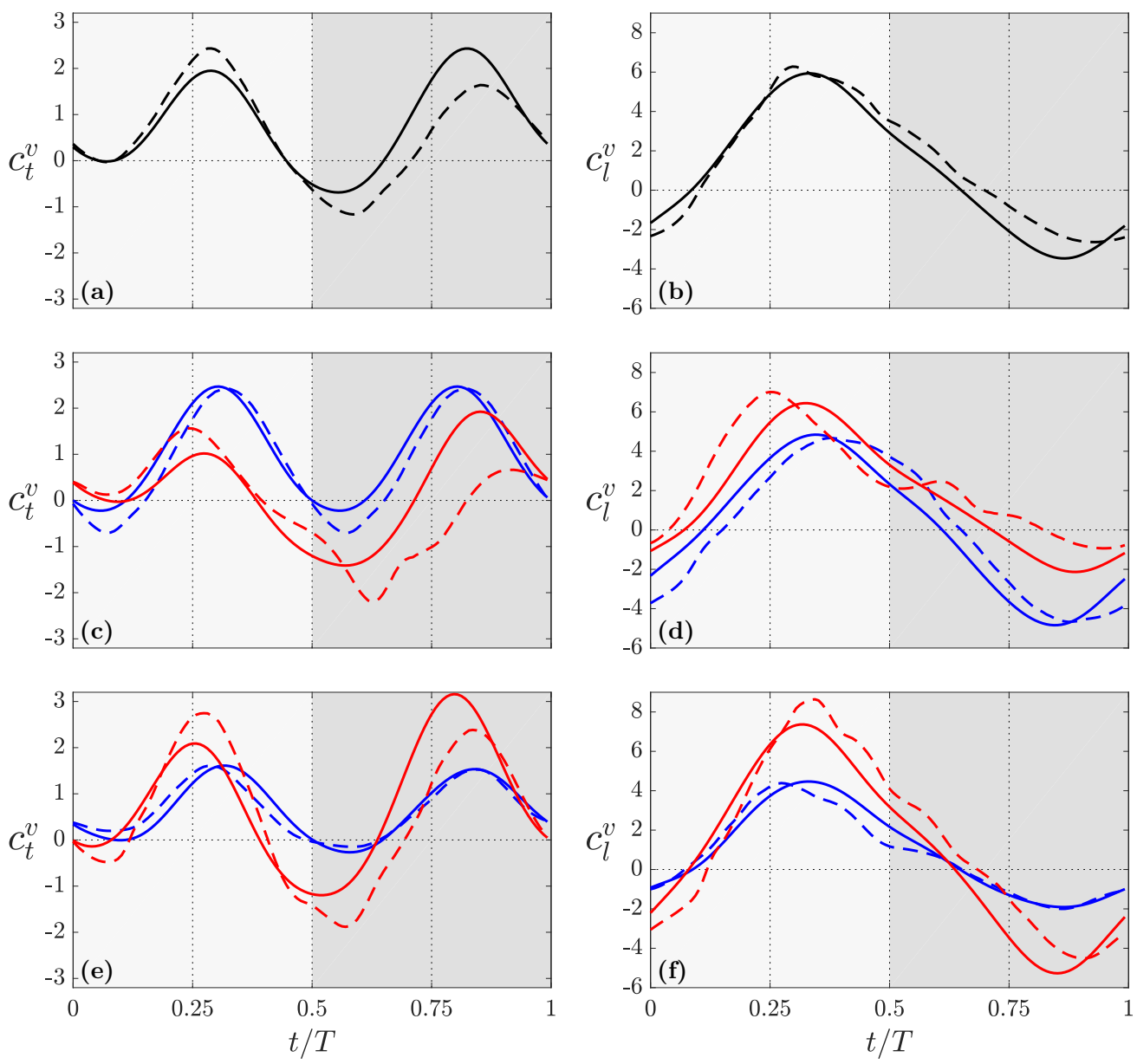

Figure 12: Chord-normal estimation of the contribution of vorticity within the flow for the selected cases. The circulation is given by the model (equation (14) ) with coefficients $G_{T}=1.85$ and $G_{R}=\pi$. a) Thrust and b) lift of case B090 (DNS --- and model - ). c) Thrust and d) lift of cases A090 (DNS --- and model - ) and C090 (DNS --- and model - ). e) Thrust and f) lift of cases B070 (DNS --- and model - ) and B110 (DNS --- and model - ). The downstroke (upstroke) is indicated by a light (dark) grey background.

the circulation without having to run a DNS or a experiment. We have tried to fit the values of $G_{T}$ and $G_{R}$ in slightly different ways (for instance, $G_{T}=1$ and fit $G_{R}$ ), but in all cases the $L_{2}$ norms of the difference in the circulation were similar as those presented in table 2. From that point of view, it could be argued that the results presented below are robust with respect to the values of these two parameters.

Figure 12 shows the goodness of the chord-normal model $\left(\vec{F}^{v}\right.$ perpendicular to the chord, modulus of $\vec{F}^{v}$ given by equations (14) and (13), $G_{T}=1.85$ and $\left.G_{R}=\pi\right)$ to predict $c_{t}^{v}$ and $c_{l}^{v}$ for the selected cases. Results for case B090 are shown in figures $12 \mathrm{a}$ and b, showing similar results to those obtained when using specific coefficients (figure 10). This is consistent with the values of the $L_{2}$ norm shown in table 2. Figures 12 and $\mathrm{d}$ show the evolution of $c_{t}^{v}$ and $c_{l}^{v}$, respectively, for cases A090 and C090 (varying the mean pitch angle). Both $c_{t}^{v}$ and $c_{l}^{v}$ are properly predicted for case A090, but the differences between the chord-normal estimation and the results obtained from the DNS are larger for case C090. These differences consist on both a small time shift and a change in the peak values. Figures 12 and $\mathrm{f}$ presents the comparison of model and DNS for B070 and B110 (varying the phase 
shift), showing a better prediction for case B070 than for case B110. Overall, the results in figure 12 suggest that the chord normal model for the vorticity within the flow works better for moderate $\theta_{m}$ and $\varphi \lesssim 90^{\circ}$.

\section{Prediction of total aerodynamic forces}

In this last section we estimate the total aerodynamic force taking into account the results presented throughout the manuscript. We discuss two different models, namely M1 and M2. While M1 introduces features based on observations made through this work, M2 is selected for comparison, based on previous works [Andersen et al., 2005, Pesavento and Wang, 2004]. Both models consider inertial effects originated from the motion of the airfoil and forces produced by the vorticity within the flow. In M1, the inertial force is the body motion term of Chang [1992], $\vec{F}^{m}$, and it is computed using (2) and the auxiliary potential given in figure 17. The force due to the vorticity within the flow is estimated as discussed in the previous section: $F^{v}$ is normal to the airfoil chord, with a modulus given by (13) and (14).

On the other hand, M2 is the model proposed by Andersen et al. [2005], but without the viscous terms. The effect of the body motion is estimated using the added mass expressions of Sedov 1965 for a flat plate hinged at $c / 4$. The contribution from the vorticity within the flow is estimated as a force perpendicular to the effective velocity $\vec{U}$, with modulus given by expressions (13) and (14).

Note that in order to minimize the differences between M1 and M2, the coefficients in (14) are $C_{T}=1.85$ and $C_{R}=\pi$ for both models. Still, there are two important differences between M1 and M2. First, the nature of the contribution from the inertial forces, which in M1 can yield a net force (i.e, $\bar{c}_{t}{ }^{m}$ and $\bar{c}_{l}{ }^{m}$ are not necessarily zero) while in M2 does not yield a net force. Second, the orientation of the contribution from the vorticity within the flow, which is one of the main observations of section 4.

Table 3 shows a quantitative comparison of the estimations of M1 and M2 in terms of the differences between mean and instantaneous values of the lift and thrust coefficients of each model and the DNS. The data in table 3 are complemented by figures 13,14 and 15, which show the instantaneous force coefficients obtained with both models and with the DNS.

Starting with the thrust, predicted mean and instantaneous values are consistently improved with M1 with respect to M2. Furthermore, $\bar{c}_{t, M 1}$ shows good agreement with the DNS results, with differences between M1 and DNS lower than 20\% of the rms of the total thrust. The estimate of mean thrust becomes less accurate for cases with $\theta_{m}=20^{\circ}$, with differences of the order of $50 \%$ of the rms of the total force. Interestingly, cases with $\varphi=70^{\circ}$ show the smallest differences in thrust for each $\theta_{m}$. This can also be appreciated in the instantaneous values of $c_{t}$ shown in figure 13 (compare the results of the third row to the other panels). As with the mean values, the estimations of the instantaneous thrust coefficients of M1 are less accurate for $\theta_{m}=20^{\circ}$. Also, when $\varphi \geq 110^{\circ}$, the instantaneous values of $c_{t}$ from M1 tend to peak earlier than the corresponding DNS values (see figures $13 \mathrm{~m}, \mathrm{n}, \mathrm{p}$ and $\mathrm{q})$.

Regarding the mean lift, the predictions of M1 are, in general, better than those of M2, except for cases B030, B050 and C030. However, for these cases the $L_{2}$ norm of the differences between M1 and the DNS are smaller than those corresponding to M2 (see also figures $14 \mathrm{~b}$, cand e). The predictions of M1 in terms of the rms of the lift coefficient improve with respect to M2 for all cases, except for A090 and A110. Cases with phase shift $\varphi<90^{\circ}$ present differences in mean lift lower than $5 \%$ of the rms of total lift and cases with higher phase shift $\varphi \geq 90^{\circ}$, lower than $20 \%$. Instantaneous values are very well captured for cases with $\theta_{m}=0^{\circ}, 10^{\circ}$ and $50 \leq \varphi \leq 110^{\circ}$ (figure 14). Again, a shift between both models and the DNS appears for the cases with higher phase shift $\left(\varphi=130^{\circ}\right)$, and errors tend to increase when the mean pitch angle is increased to $\theta_{m}=20^{\circ}$. For the latter, the peak in the lift during the downstroke is consistently underestimated by both models. Finally, for the symmetric cases $\left(\theta_{m}=0^{\circ}\right)$, the estimated mean lift is zero in both models (as expected). The differences that 

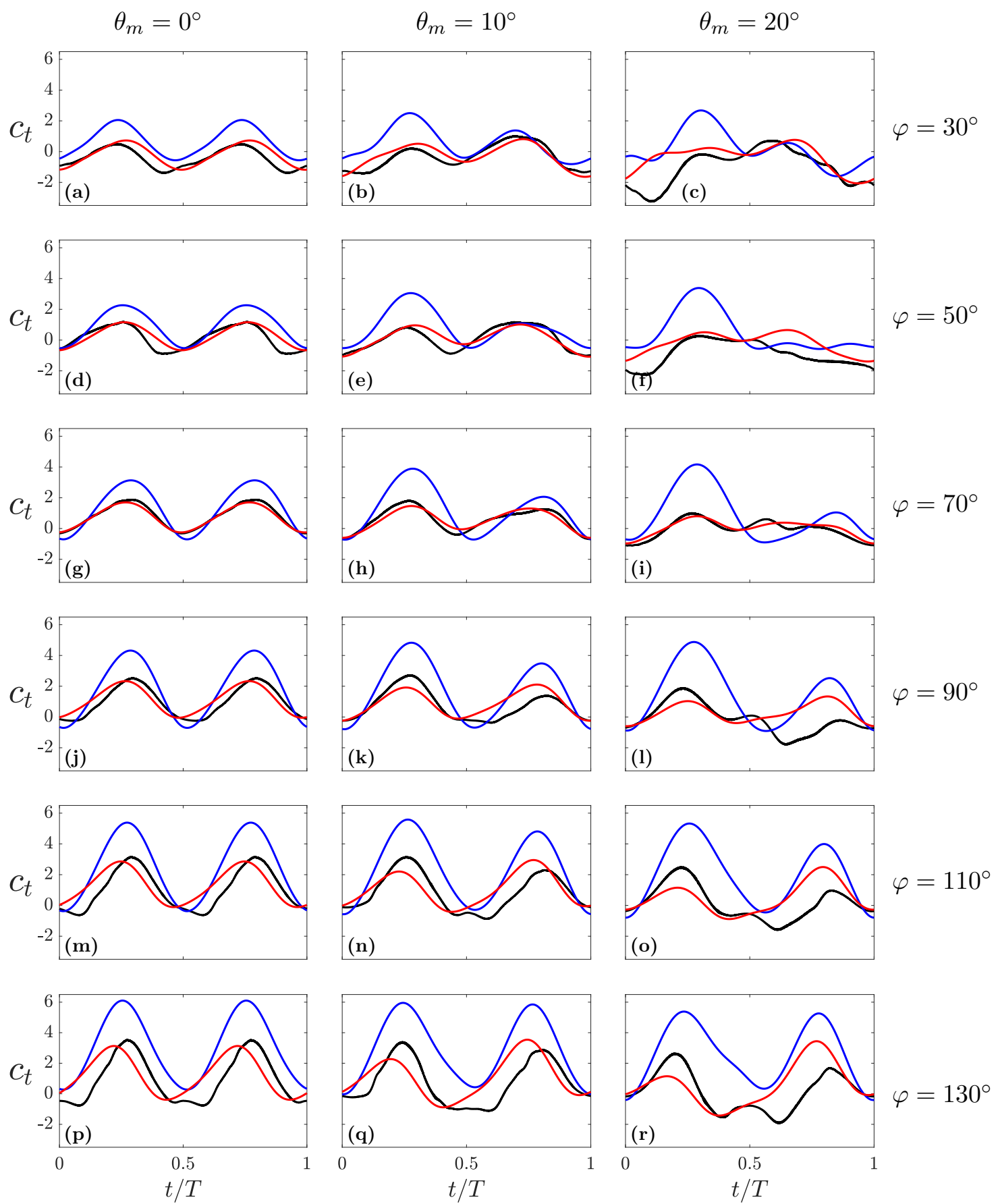

Figure 13: Total thrust obtained from the DNS ( - ) together with the estimations with M1 ( ) and M2 ( - ). 


\begin{tabular}{|l|rr|rr|rr|rr|} 
& \multicolumn{2}{|c|}{$\bar{c}_{t, M \#}-\bar{c}_{t, D N S}$} & \multicolumn{2}{|c|}{$\left\|c_{t, M \#}-c_{t, D N S}\right\|_{2}$} & \multicolumn{2}{c|}{$\bar{c}_{l, M \#}-\bar{c}_{l, D N S}$} & \multicolumn{1}{c|}{$\left\|c_{l, M \#}-c_{l, D N S}\right\|_{2}$} \\
\hline Case & M1 & M2 & M1 & M2 & \multicolumn{1}{c|}{ M1 } & M2 & M1 \\
A030 & 0.226 & 1.109 & 0.444 & 1.168 & 0.004 & 0.004 & 1.426 & 2.428 \\
A050 & 0.106 & 0.785 & 0.402 & 0.994 & 0.000 & 0.000 & 0.689 & 1.793 \\
A070 & -0.066 & 0.513 & 0.131 & 0.808 & -0.000 & -0.000 & 0.268 & 1.004 \\
A090 & 0.116 & 0.835 & 0.471 & 1.173 & 0.000 & -0.000 & 0.796 & 0.316 \\
A110 & 0.246 & 1.400 & 0.876 & 1.654 & -0.000 & -0.000 & 1.245 & 1.040 \\
A130 & 0.287 & 2.096 & 1.181 & 2.221 & 0.005 & 0.005 & 1.896 & 2.235 \\
B030 & 0.065 & 0.864 & 0.395 & 1.196 & 0.102 & -0.011 & 1.503 & 2.368 \\
B050 & 0.042 & 0.724 & 0.355 & 1.195 & -0.108 & -0.043 & 1.038 & 1.953 \\
B070 & -0.025 & 0.627 & 0.268 & 1.131 & 0.010 & 0.242 & 0.826 & 1.700 \\
B090 & 0.166 & 0.995 & 0.620 & 1.377 & 0.557 & 0.913 & 0.699 & 1.182 \\
B110 & 0.209 & 1.467 & 0.852 & 1.766 & 0.597 & 1.014 & 1.107 & 1.404 \\
B130 & 0.311 & 2.179 & 1.181 & 2.396 & 0.805 & 1.209 & 1.934 & 2.511 \\
C030 & 0.628 & 1.176 & 1.077 & 1.717 & 0.984 & 0.732 & 2.593 & 2.859 \\
C050 & 0.693 & 1.358 & 0.874 & 1.683 & 1.345 & 1.388 & 2.118 & 2.427 \\
C070 & 0.094 & 0.926 & 0.223 & 1.647 & 0.307 & 0.637 & 1.409 & 2.179 \\
C090 & 0.432 & 1.544 & 1.075 & 2.026 & 1.032 & 1.587 & 1.617 & 2.400 \\
C110 & 0.336 & 1.861 & 1.136 & 2.271 & 1.240 & 1.919 & 1.569 & 2.415 \\
C130 & 0.460 & 2.474 & 1.410 & 2.894 & 1.654 & 2.337 & 2.245 & 3.166
\end{tabular}

Table 3: Mean and $L_{2}$ norm of the difference between an estimation of the thrust and lift and the value obtained from the DNS. The models presented correspond to the estimation proposed in the present work (M1) and an estimation taken from the literature (M2, see Andersen et al., 2005).

appear in cases A030 and A130 between the models and the DNS arise from the averaging process of the DNS data, due to the aperiodic nature of these cases.

The different behavior of the models in the prediction of lift and thrust coefficients are in part due to the ability of the models to predict properly the orientation of the aerodynamic force. In order to compare the two models and the DNS without taking into account this effect, figure 15 shows the total aerodynamic force coefficient, defined as

$$
c_{f}=\sqrt{c_{l}^{2}+c_{t}^{2}} .
$$

It can be observed in figure 15 that the agreement between both models and the DNS for $\theta_{m}=0^{\circ}, 10^{\circ}$ and $50^{\circ} \lesssim \varphi \lesssim 110^{\circ}$ is reasonably good. For $\varphi=30^{\circ}$ and $\theta_{m}=0^{\circ}, 10^{\circ}$, the amplitude of $c_{f}$ from the DNS is larger than both models. For $\varphi=130^{\circ}$ and $\theta_{m}=0^{\circ}, 10^{\circ}$, the magnitudes of the peaks of $c_{f}$ are reasonably captured, but the models are shifted in time with respect the DNS data. For $\theta_{m}=20^{\circ}$, independently of the value of $\varphi$, the peak of $c_{f}$ during the downstroke is underestimated in both models, which suggests that the model used for the circulation (namely equation 14) might not be properly capturing the development and evolution of the LEV for these high angle of attack cases. Note also that the differences between M1 and M2 in $c_{f}$ and $c_{l}$ (figures 14 and 15) are less apparent than in $c_{t}$ in figure 13. This suggests that the chord normal orientation of the contribution of the vorticity within the flow is more important for the prediction of the thrust.

Finally, note that the model proposed here has some limitations. As discussed in section $4, \vec{F}^{v}$ is approximately normal to the chord of the airfoil when the LEV is strong enough, and even in that case there is a small component of $\vec{F}^{v}$ tangential to the airfoil chord. This tangential component is unimportant for the cases discussed here, but it can be significant in some cases, like the pure heaving case. Indeed, for a pure heaving case at zero pitch angle, M1 predicts $c_{t}=0$ (since both vorticity within the flow and body motion components result in forces perpendicular to the chord). This means 

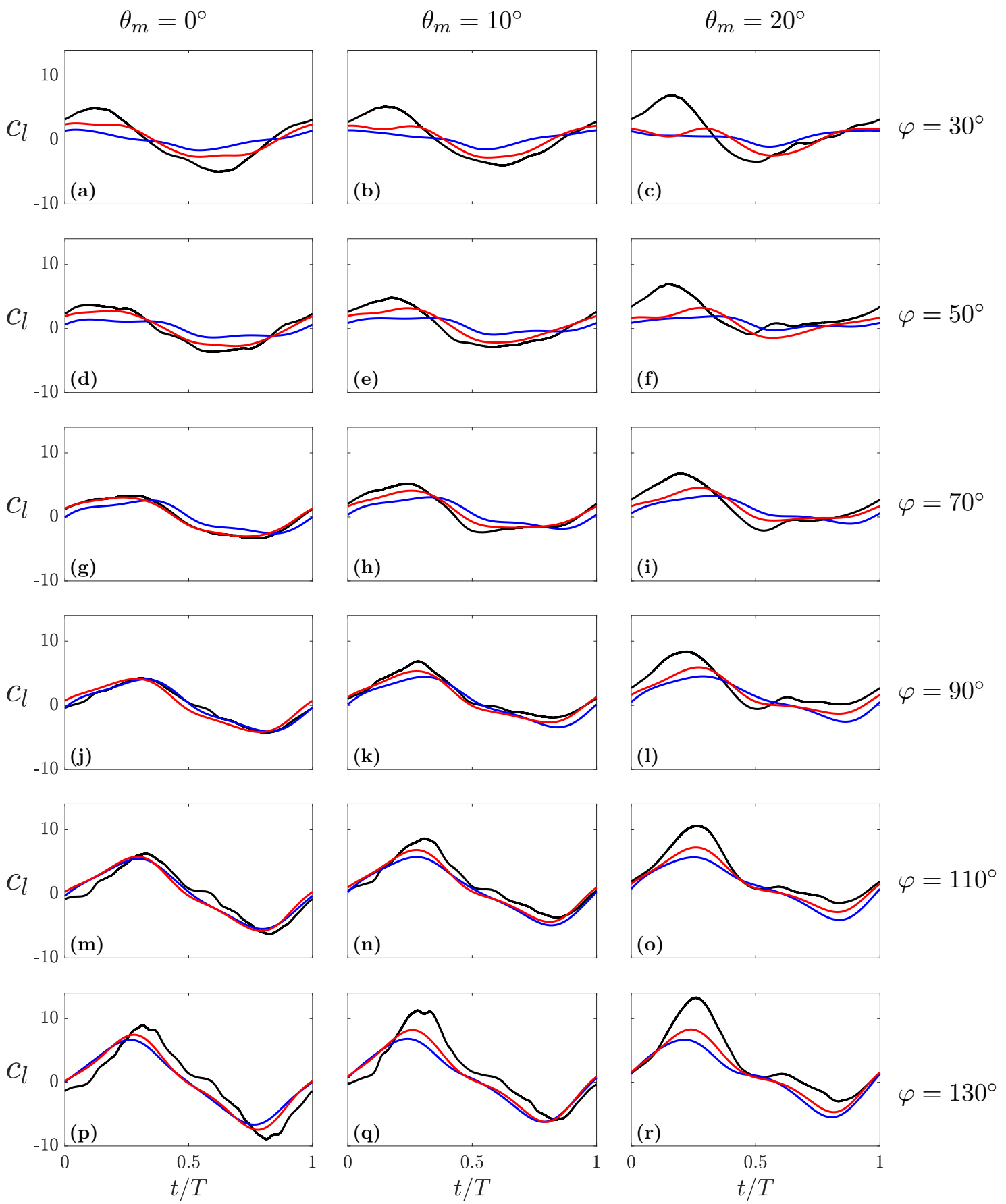

Figure 14: Total lift obtained from the DNS ( - ) together with the estimations with M1 ( - ) and M2 ( - ). 
that further work is needed to widen the range of applicability of M1, in order to include pure heaving cases.

\section{Conclusions}

In this work we have generated and analyzed a database of DNS of heaving and pitching airfoils, with large amplitude motions, moderate Reynolds numbers and reduced frequencies of order $O(1)$. In order to analyze the effect of the mean pitch angle and the phase shift, we have varied these parameters in the ranges $\theta_{m} \in\left[0^{\circ}, 20^{\circ}\right]$ and $\varphi \in\left[30^{\circ}, 130^{\circ}\right]$. In terms of the lift and thrust obtained by these configurations, we observe that as $\theta_{m}$ increases the mean lift increases and the mean thrust decreases with little variations in the amplitude of the instantaneous fluctuations. This is consistent with a change in the orientation of the force when $\theta_{m}$ varies, analogous to a change in the stroke plane. On the other hand, the effect of the phase shift is less intuitive: the net lift and thrust increase with $\varphi$ for $\varphi \lesssim 110^{\circ}$, as well as the amplitude of their fluctuations. As a consequence, in our database the maximum propulsion efficiency is obtained for $\varphi=90^{\circ}$ and the maximum force is obtained for $\varphi=110^{\circ}$. This is consistent with previous investigations Anderson et al., 1998, Ramamurti and Sandberg, 2001.

Subsequently, we have decomposed the total aerodynamic force following Chang [1992]. We observe that for the considered kinematics, the contributions from the vorticity within the flow and body motion are comparable, while the surface vorticity contribution is significantly smaller. The contribution of vorticity within the flow is influenced only by the vortices in the vicinity of the airfoil and is roughly perpendicular to the chord. Note that since vorticity within the flow is the main contributor to the net aerodynamic forces, the effects of $\varphi$ and $\theta_{m}$ are the same as discussed in the previous paragraph for the total forces. Conversely, for a given value of $\theta_{m}$, the net contribution from body motion to the thrust and lift increases monotonically with $\varphi$.

Finally, based on the observations made from the force decomposition analysis, we have proposed and tested a reduced order model for the aerodynamic forces. We compute the body motion contribution directly from Chang [1992], since it only depends on geometric characteristics of the airfoil (the auxiliary potentials) and on the kinematics. We model the vorticity within the flow contribution as a force perpendicular to the chord and whose modulus is proportional to the circulation of the airfoil. This circulation is calculated with the model of Pesavento and Wang 2004. The only parameters that need fitting are the constants $G_{T}$ and $G_{R}$ appearing in the model for the circulation. Our results show that these two constants can be fixed for the whole database keeping a reasonable error in the force estimation, which suggests that the model is able to take into account the kinematic parameters that are varied in this study $\left(\theta_{m}\right.$ and $\left.\varphi\right)$. Overall, the model is able to predict mean thrust and lift with errors smaller than $20 \%$ of the rms of the corresponding forces for $\theta_{m}=0^{\circ}$ and $10^{\circ}$. From the point of view of the instantaneous forces the agreement between the model and the DNS data is very satisfactory for $\theta_{m}=0^{\circ}$ and $10^{\circ}$, and $50^{\circ} \lesssim \varphi \lesssim 110^{\circ}$. For $\theta_{m}=20^{\circ}$, the instantaneous forces suggest that the error in the estimation of the mean forces is due to the underestimation of the intensity of the LEV during the downstroke. The predictions of the proposed model are compared to those of a similar model from the literature, showing a noticeable improvement on the prediction of the mean thrust, and a smaller improvement on the prediction of mean lift and the instantaneous force coefficients.

The authors acknowledge the support received by the Spanish Ministry of Economy and Competitiveness through grant TRA2013-41103-P. This grant includes FEDER funding. 

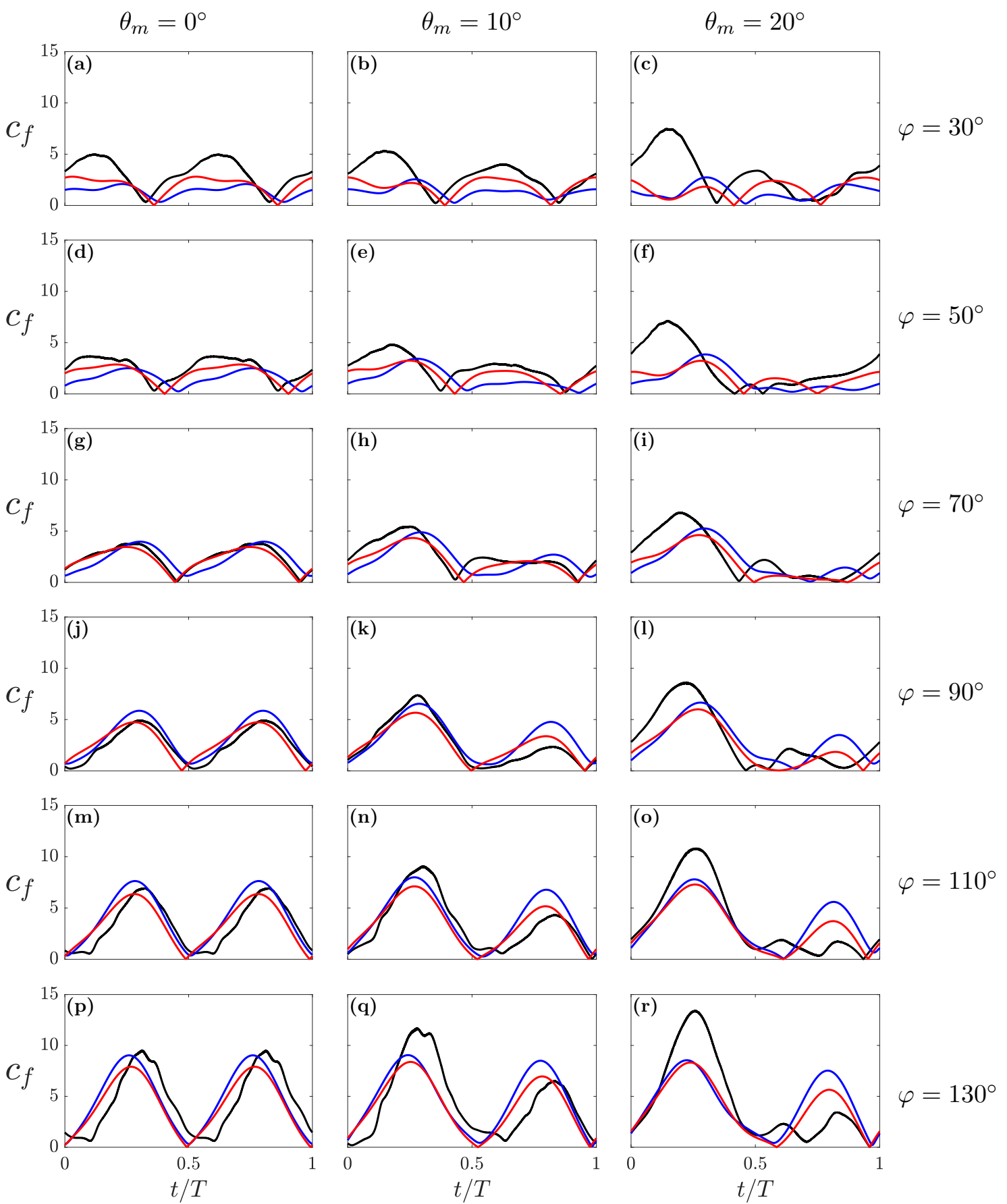

Figure 15: Total aerodynamic force modulus obtained from the DNS ( - ) together with the estimations with M1 ( - ) and M2 ( - ). 


\section{References}

A. Andersen, U. Pesavento, and Z. J. Wang. Unsteady aerodynamics of fluttering and tumbling plates. J. Fluid Mech., 541:65-90, 2005.

J.M. Anderson, K. Streitlien, K. S. Barret, and M. S. Triantafyllou. Oscillating foils of high propulsive efficiency. J. Fluid Mech., 360:41-72, 1998.

R. Ansari, R. Zbikowski, and K. Knowles. Aerodynamic modelling of insect-like flapping flight for micro air vehicles. Prog. Aerospace Sci., 42:129-172, 2006.

M. A. Ashraf, J. Young, and J. C. S. Lai. Reynolds number, thickness and camber effects on flapping airfoil propulsion. J. Fluids Struct., 27:145-160, 2011.

Y. S. Baik, L. P. Bernal, K. Granlund, and M. V. Ol. Unsteady force generation and vortex dynamics of pitching and plunging aerofoils. J. Fluid Mech., 709:37-68, 2012.

L. W. Carr. Progress in analysis and prediction of dynamic stall. J Aircraft, 25(1):6-17, 1988.

C.-C. Chang. Potential flow and forces for incompressible viscous flow. In Proc. R. Soc. Lond. A, volume 437, pages 517-525, 1992.

J. Choi, T. Colonius, and D. R. Williams. Surging and plunging oscillations of an airfoil at low Reynolds number. J. Fluid Mech., 763:237-253, 2015.

M. H. Dickinson, F.-O. Lehmann, and S. P. Sane. Wing rotation and the aerodynamic basis of insect flight. Science, 284(5422):1954-1960, 1999.

C. P. Ellington, C. Van Den Berg, A. P. Willmott, and A.L.R. Thomas. Leading-edge vortices in insect flight. Nature, 384:626-630, 1996.

Y. C. Fung. An introduction to the theory of aeroelasticity. Courier Corporation, 2002.

K. D. Jones and M. F. Platzer. Numerical computation of flapping-wing propulsion and power extraction. AIAA paper AIAA-97-0826, 1997.

G. C. Lewin and H. Haj-Hariri. Modelling thrust generation of a two-dimensional heaving airfoil in a viscous flow. J. Fluid Mech., 492:339-362, 2003.

L. N. Long and T. E. Fritz. Object-oriented unsteady vortex lattice method for flapping flight. $J$. Aircraft, 41(6):1275-1290, 2004.

K. B. Lua, T. T. Lim, K. S. Yeo, and G. Y. Oo. Wake-structure formation of a heaving two-dimensional elliptic airfoil. AIAA J., 45:1571-1583, 2007.

A. Martín-Alcántara, R. Fernandez-Feria, and E. Sanmiguel-Rojas. Vortex flow structures and interactions for the optimum thrust efficiency of a heaving airfoil at different mean angles of attack. Phys. Fluids, 27(7):073602, 2015.

R. Mittal, H. Dong, M. Bozkurttas, F.M. Najjar, A. Vargas, and A. Von Loebbecke. A versatile sharp interface immersed boundary method for incompressible flows with complex boundaries. J. Comput. Phys., 227(10):4825-4852, 2008.

M. Moriche. A numerical study on the aerodynamic forces and the wake stability of flapping flight at low Reynolds number. PhD thesis, Universidad Carlos III de Madrid, 2017. 
M. Moriche, O. Flores, and M. García-Villalba. Generation of thrust and lift with airfoils in plunging and pitching motion. In J. Phys.: Conf. Ser., volume 574, page 012163, 2015.

M. Moriche, O. Flores, and M. García-Villalba. Three-dimensional instabilities in the wake of a flapping wing at low Reynolds number. Int. J. Heat Fluid Flow, 62 A:44 - 55, 2016.

F. Noca, D. Shiels, and D. Jeon. A comparison of methods for evaluating time-dependent fluid dynamic forces on bodies, using only velocity fields and their derivatives. J. Fluids Struct., 13(5):551-578, 1999.

U. Pesavento and Z.J. Wang. Falling paper: Navier-Stokes solutions, model of fluid forces, and center of mass elevation. Phys. Rev. Lett., 93(14):144501, 2004.

M.F. Platzer, K.D. Jones, J. Young, and J.C.S. Lai. Flapping-wing aerodynamics: Progress and challenges. AIAA J., 46(9):2136-2149, 2008.

R. Ramamurti and W. Sandberg. Simulation of flow about flapping airfoils using finite element incompressible flow solver. AIAA J., 39(2):253-260, 2001.

D. A. Read, F. S. Hover, and M. S. Triantafyllou. Forces on oscillating foils for propulsion and maneuvering. J. Fluids Struct., 17(1):163-183, 2003.

K.V. Rozhdestvensky and V.A. Ryzhov. Aerohydrodynamics of flapping-wing propulsors. Prog. Aerospace Sci., 39:585-633, 2003.

L. I. Sedov. Two-dimensional problems in hydrodynamics and aerodynamics. Interscience Publishers, 1965.

W. Shyy, H. Aono, Chimakurthi S.K., P. Trizila, C.-K. Kang, C.E.S. Cesnik, and H. Liu. Recent progress in flapping wing aerodynamics and aeroelasticity. Prog. Aerospace Sci., 46:284-327, 2010.

W. Shyy, H. Aono, C.-K. Kang, and H. Liu. An introduction to flapping wing aerodynamics. Cambridge Univ. Press, 2013.

H. E. Taha, M. R. Hajj, and A. H. Nayfeh. Flight dynamics and control of flapping-wing MAVs: a review. Nonlinear Dyn., 70(2):907-939, 2012.

H. E. Taha, M. R. Hajj, and P. S. Beran. State-space representation of the unsteady aerodynamics of flapping flight. Aerosp. Sci. Technol., 34:1-11, 2014.

T. Theodorsen. General theory of aerodynamic instability and the mechanism of flutter. Technical report, NACA, 1949.

M. Uhlmann. An immersed boundary method with direct forcing for the simulation of particulate flows. J. Comput. Phys., 209(2):448-476, 2005.

K. D. von Ellenrieder, K. Parker, and J. Soria. Fluid mechanics of flapping wings. Exp. Thermal Fluid Sci., 32:1578-1589, 2008.

H. Wagner. Über die entstehung des dynamischen auftriebes von tragflügeln. ZAMM-Z. Angew. Math. Me., 5(1):17-35, 1925.

S. Wang, X. Zhang, G. He, and T. Liu. Evaluation of lift formulas applied to low-Reynolds-number unsteady flows. AIAA J., 53(1):161-175, 2015.

Z.J. Wang. Vortex shedding and frequency selection in flapping flight. J. Fluid Mech., 410:323-341, 2000. 

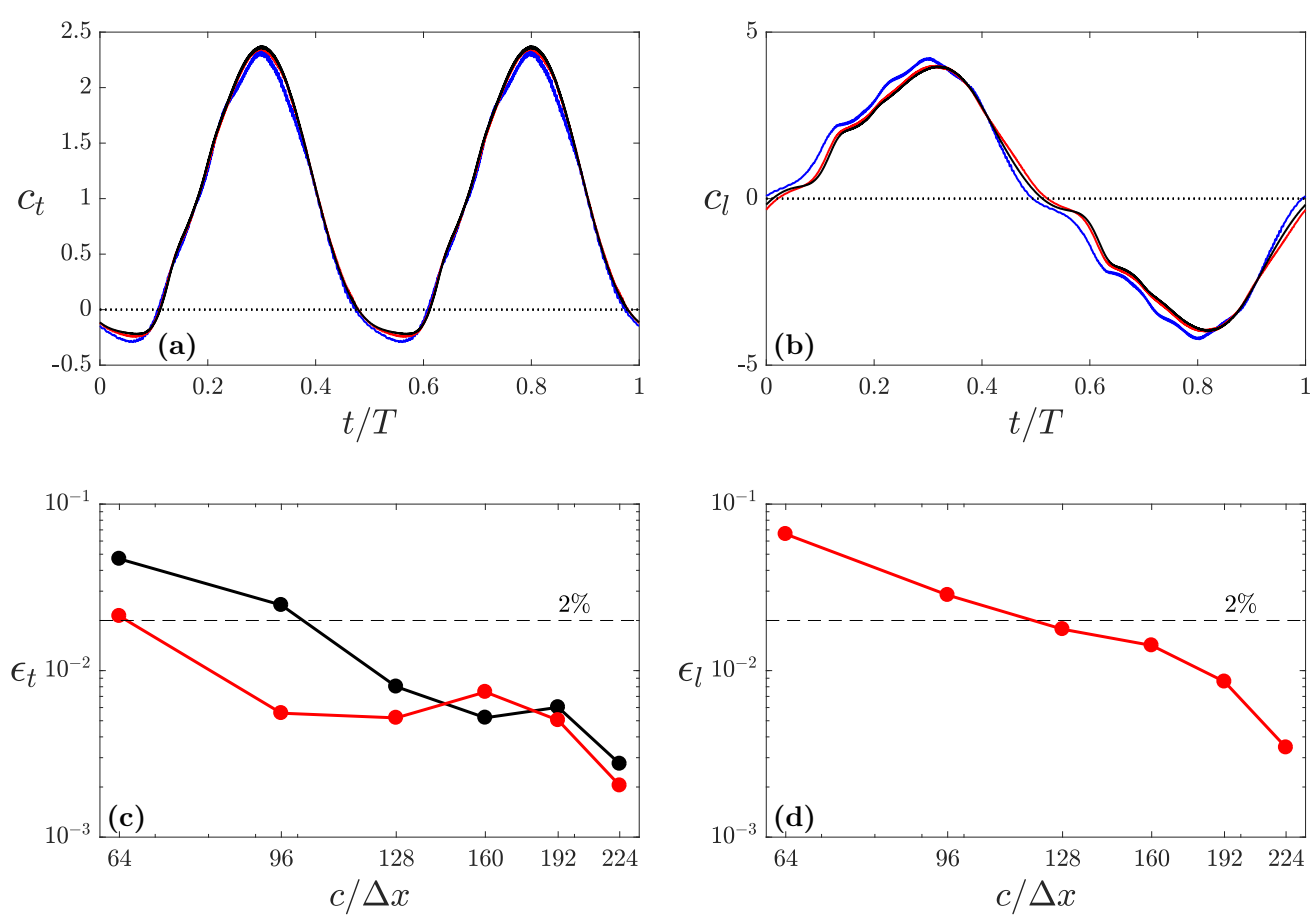

Figure 16: Evolution of a) thrust and b) lift for cases with $c / \Delta x=64(-), c / \Delta x=128(-)$ and $c / \Delta x=256(-)$. Errors obtained in mean $(\bullet)$ ) and $\mathrm{rms}(\bullet)$ values of $\mathrm{c}$ ) thrust and d) lift of all the simulations.

Z. Wei and Z. C. Zheng. Mechanisms of wake deflection angle change behind a heaving airfoil. $J$. Fluids Struct., 48:1-13, 2014.

A. Widmann and C. Tropea. Parameters influencing vortex growth and detachment on unsteady aerodynamic profiles. J. Fluid Mech., 773:432-459, 2015.

J. Z. Wu, Z. L. Pan, and X. Y. Lu. Unsteady fluid-dynamic force solely in terms of control-surface integral. Phys. Fluids, 17(9):098102, 2005.

\section{A Grid refinement study}

In this section we present the grid refinement study carried out to select the resolution used in the simulations. In order to save computational time, a smaller computational domain has been employed in the grid refinement study, namely, $12 c \times 8 c$ in the streamwise and vertical directions, respectively. The parameters of the case under consideration correspond to case A090 reported in table 1. This is a case in which mean thrust is produced while the mean lift is zero. We have performed seven simulations varying the resolution from $c / \Delta x=64$ to $c / \Delta x=256$ and the time step $\Delta t$ is set accordingly to keep a CFL number lower than 0.2 . We study the convergence of the aerodynamic forces when increasing the resolution, and we use as a reference data the results of the case with the highest resolution $(c / \Delta x=256)$. The time evolution of the thrust and lift coefficients of three of the simulations are reported in figure $16 \mathrm{a}$ and $\mathrm{b}$. While some deviations are observed for the resolution $c / \Delta x=64$ with respect to the reference case, the results of the simulation with resolution $c / \Delta x=128$ are very close to those of the reference case. In order to quantify the differences we define the errors in the mean 
and rms of the forces

$$
\begin{aligned}
\epsilon_{t}^{\text {mean }}(r) & =\frac{\left|\bar{c}_{t r}-\bar{c}_{t 256}\right|}{c_{t 256}^{\prime}}, \\
\epsilon_{t}^{\mathrm{rms}}(r) & =\frac{\left|c_{t r}^{\prime}-c_{t 256}^{\prime}\right|}{c_{t 256}^{\prime}}, \\
\epsilon_{l}^{\text {mean }}(r) & =\frac{\left|\bar{c}_{l r}\right|}{c_{l 256}^{\prime}}, \\
\epsilon_{l}^{\mathrm{rms}}(r) & =\frac{\left|c_{l r}^{\prime}-c_{l 256}^{\prime}\right|}{c_{l 256}^{\prime}},
\end{aligned}
$$

where $r$ is the resolution of each case. Since the case under consideration produces no lift, the definition of $\epsilon_{l}^{\text {mean }}(r)$ does not need to have a reference mean lift. Figures 16; and d show the errors as a function of the resolution. Note that $\epsilon_{l}^{\text {mean }}(r)$ is not shown in figure $16 \mathrm{~d}$ since it is smaller than $10^{-4}$ for all the cases. In general the errors decrease with increasing resolution. Taking into account a compromise between the computational cost and the accuracy of the results, we have decided to use a resolution of $c / \Delta x=128$ in our calculations. With this resolution the errors for both mean and rms of the forces are smaller than $2 \%$ as shown in the figure.

\section{B Calculation of auxiliary potential functions}

The force decomposition algorithm used in this work was first introduced by Chang [1992 and later used by Martín-Alcántara et al. 2015]. The algorithm requires two auxiliary potential functions $\phi_{x}$ and $\phi_{z}$ (see equation 2), which are defined as

$$
\begin{aligned}
\nabla^{2} \phi_{x} & =0 \\
\nabla \phi_{x} \cdot \vec{n} & =-\vec{n} \cdot \vec{e}_{x} U_{\infty} \\
\phi_{x} & \rightarrow 0 \\
\nabla^{2} \phi_{z} & =0 \\
\nabla \phi_{z} \cdot \vec{n} & =-\vec{n} \cdot \vec{e}_{z} U_{\infty} \\
\phi_{z} & \rightarrow 0
\end{aligned}
$$$$
\text { At the body surface }
$$$$
\text { At infinity, }
$$

where $\vec{n}$ is a unitary vector normal to the surface of the airfoil pointing towards the fluid.

The auxiliary potential functions $\phi_{x}$ and $\phi_{z}$ are needed at every time step in which the force decomposition is to be applied. These potentials depend only on the airfoil geometry and on the direction of the free stream velocity, not on the heaving and pitching velocities and accelerations. Note that the dependency with the orientation is linear, so that it is possible to show that for an arbitrary direction $\vec{\alpha}=\alpha_{1} \vec{e}_{x}+\alpha_{2} \vec{e}_{z}$, the corresponding potential $\phi_{\alpha}$ satisfies

$$
\begin{aligned}
\nabla^{2} \phi_{\alpha} & =0 \\
\nabla \phi_{\alpha} \cdot \vec{n} & =-\vec{n} \cdot \vec{\alpha} U_{\infty} \\
\phi_{\alpha} & \rightarrow 0
\end{aligned}
$$

At the body surface

At infinity.

Hence this potential can be computed as $\phi_{\alpha}=\alpha_{1} \phi_{x}+\alpha_{2} \phi_{z}$.

The linearity with the orientation eliminates the problem of having to compute the auxiliary potential functions at different time instants. Instead, the auxiliary potential functions are computed 

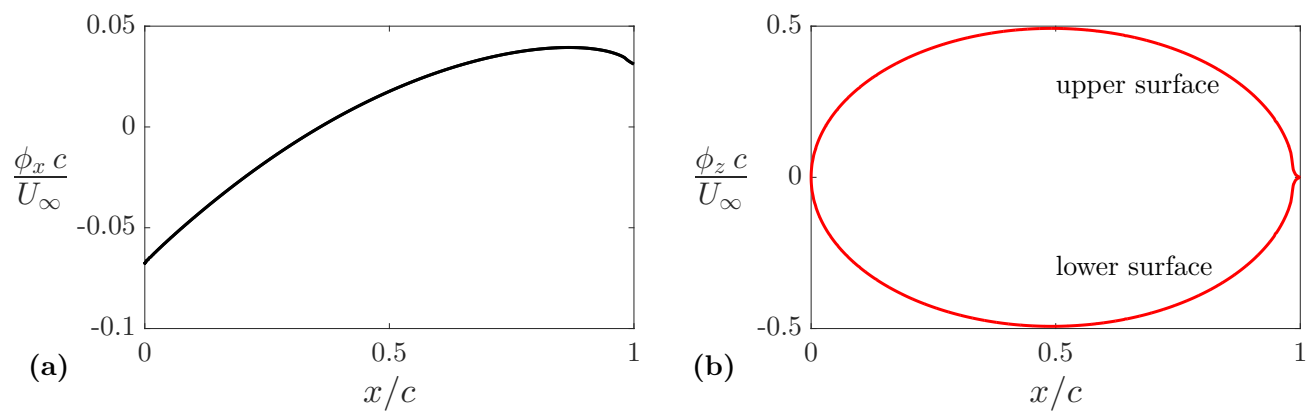

Figure 17: Auxiliary potentials a) $\phi_{x}$ and b) $\phi_{z}$ for a NACA 0012 airfoil at $\theta=0^{\circ}$.

for a reference position of the airfoil, and then rotated (using 20) and translated to the position of the airfoil at each time instant.

Finally, it should be noted that the auxiliary potential functions $\phi_{x}$ and $\phi_{z}$ only have analytical solutions for simple geometries, like ellipses Martín-Alcántara et al., 2015. For other geometries, like the NACA 0012 considered in the present work, they need to be computed numerically. This is done using the sharp interface method of Mittal et al. 2008, which is based on an immersed boundary formulation where normal derivatives on the solid boundary are imposed by using image and ghost points. The implementation of the solver has been validated computing the potential function for ellipses of different aspect ratios, and comparing the numerical results to the analytical solutions. For the computations of the potential functions at the reference orientation of the NACA 0012 airfoils (shown in figure 17), we have employed a computational domain of $30 c \times 30 c$. This domain has been discretized using $6072 \times 6072$ grid points in the chordwise and vertical directions, respectively. The translated and rotated potential functions are then interpolated with a linear interpolator to the collocation points where the integrals of (2) are computed.

In order to compute the body motion contribution $\vec{F}^{m}$ to the total aerodynamic force, we present the value of the potentials $\phi_{x}$ and $\phi_{z}$ at the surface of a NACA 0012 airfoil in the reference position (figures $17 \mathrm{a}$ and $\mathrm{b}$ ). 